\title{
Study on the Role of the Inclusion Complexes with 2- Hydroxypropyl- $\beta$-cyclodextrin for Oral Administration of Amiodarone
}

\author{
Andreea Creteanu $\left(\mathbb{D},{ }^{1}\right.$ Daniela Pamfil $\left(\mathbb{D},{ }^{2}\right.$ Cornelia Vasile $\mathbb{D},{ }^{2}$ Gladiola Tantaru $\left(\mathbb{D},{ }^{3}\right.$ \\ Cristina Mihaela Ghiciuc $\left(\mathbb{D},{ }^{4}\right.$ Lacramioara Ochiuz $\left(\mathbb{D},{ }^{1}\right.$ Alina Ghilan $(\mathbb{D})^{2}$ \\ and Ana Maria Macsim ${ }_{(\mathbb{D})}^{2}$ \\ ${ }^{1}$ Department of Pharmaceutical Technology, Faculty of Pharmacy, "Grigore T. Popa” University of Medicine and Pharmacy of Iasi, \\ 16, Universitatii Str., 700115 Iasi, Romania \\ ${ }^{2}$ Physical Chemistry of Polymers Department, Petru Poni Institute of Macromolecular Chemistry, 41A Gr. Ghica Voda Alley, \\ RO700487, Iasi, Romania \\ ${ }^{3}$ Department of Analytical Chemistry, Faculty of Pharmacy, "Grigore T. Popa" University of Medicine and Pharmacy, 16, \\ University Str., 700115 Iasi, Romania \\ ${ }^{4}$ Department of Pharmacology, Faculty of Medicine, "Grigore T. Popa” University of Medicine and Pharmacy, 16, University Str., \\ 700115 Iasi, Romania
}

Correspondence should be addressed to Andreea Creteanu; acreteanu@gmail.com and Cornelia Vasile; cvasile@icmpp.ro

Andreea Creteanu, Daniela Pamfil, Cornelia Vasile, Gladiola Tantaru, Cristina Mihaela Ghiciuc, Lacramioara Ochiuz, Alina Ghilan, and Ana Maria Macsim contributed equally to this work.

Received 24 July 2019; Revised 18 September 2019; Accepted 25 September 2019; Published 28 November 2019

Academic Editor: Qinglin Wu

Copyright (C) 2019 Andreea Creteanu et al. This is an open access article distributed under the Creative Commons Attribution License, which permits unrestricted use, distribution, and reproduction in any medium, provided the original work is properly cited.

\begin{abstract}
The aim of this study was to improve the solubility of amiodarone hydrochloride (AMD) and the drug release using its inclusion complexes with 2-hydroxypropyl- $\beta$-cyclodextrin (HP- $\beta$-CD). The inclusion complexes were prepared by coprecipitation and freeze-drying. The solubility enhancement of AMD/HP- $\beta$-CD inclusion complexes by $4-22$ times was evaluated by the phase solubility method. The inclusion complexes were studied both in solution and in solid state by spectroscopic methods, dynamic light scattering (DLS) and zeta potential analysis, SEM, and DSC. The formulations of AMD/HP- $\beta$-CD inclusion complexes both as powdered form and as matrix tablets showed superior pharmacokinetic performance in improving loading and release properties in respect of those of the insoluble AMD drug. In vitro kinetic study reveals a complex mechanism of release occurring in three steps: the first one being attributed to a burst effect and the other two to different bonding existing in inclusion complexes. An in vivo test on matrix tablets containing Kollidon ${ }^{\circledR}$ and chitosan also reveals a multiple (at least two) peaks release diagram because of both structures of the inclusion complexes and also of different sites of absorption in biological media (digestive tract).
\end{abstract}

\section{Introduction}

Amiodarone hydrochloride (AMD) is a highly effective antiarrhythmic drug used in the management of severe ventricular and supraventricular arrhythmias [1]. It is considered a class II antiarrhythmic drug according to Vaughan Williams' classification, but it possesses multiple electrophysiological effects (the characteristics of all four Vaughan Williams classes).
Although it generates pulmonary and hepatic toxicity and thyroid dysfunction, AMD remained the most efficient drug for the treatment of a wide variety of arrhythmias because it has demonstrated high clinical efficacy including for those complicated with Wolff-Parkinson-White syndrome or refractory to adequate doses of other antiarrhythmic drugs [2, 3].

The use of AMD, a highly lipophilic drug, is complicated by its complex pharmacokinetics [4]. The main therapeutic 
disadvantage of AMD is its low oral bioavailability and low water solubility; therefore, intravenously administered amiodarone is preferred in the acute treatment. The absorption of orally administrated AMD is slow and variable, with peak plasma concentrations that occur at 2 to 7 hours following a single oral dose. Oral bioavailability is extremely variable and ranges from 22 to $86 \%$. However, if AMD is ingested with fatty foods, the rate and extent of absorption are increased [5]. The plasma levels resulting from orally administered AMD vary considerably between individuals, while efficacy has been observed for AMD plasma concentrations as low as $0.1 \mu \mathrm{g} / \mathrm{mL}$, other studies showed therapeutic efficacy for AMD plasma concentration of $1-2.5 \mu \mathrm{g} / \mathrm{mL}[6,7]$. The low bioavailability may be related to intestinal wall metabolism mediated by CYP3A4 enzyme and gastrointestinal excretion mediated by P-glycoprotein. After absorption, AMD undergoes extensive enterohepatic circulation before being distributed to the central compartment and tissues. The maximum time $\left(t_{\max }\right)$ from oral intake to reaching peak plasma concentration $\left(C_{\max }\right)$ after a single-dose administration ranges from 2 to $10 \mathrm{~h}$. AMD is eliminated primarily by hepatic metabolism and biliary excretion.

$\mathrm{N}$-Desethylamiodarone is the CYP3A4 metabolite of amiodarone identified in plasma, which also suppressed ventricular arrhythmias [6,7]. The low water solubility of AMD extensive first pass effect might be other reasons for its large interindividual variation in systemic bioavailability. Moreover, AMD presents poor dissolution characteristics, which is one of the main factors limiting its gastrointestinal absorption. As it was mentioned, due to its slow dissolution rate and high membrane permeability, AMD is classified as a class II drug based on the Biopharmaceutical Classification System $[8,9]$.

Low solubility affects the pharmacokinetic properties of the active compounds; therefore, some solutions have been proposed, as complexation or conjugation with cyclodextrin as a method to optimize the therapeutic performance of the insoluble drugs including AMD [10-13].

Cyclodextrins (CDs) are cyclic oligosaccharides containing at least $6 \mathrm{D}-(+)$ glucopyranose units attached by $\alpha-1,4-$ glycosidic bonds. They contain a hydrophobic cavity in which the active compound is internalized forming an inclusion complex with the drug. Both inclusion complex formation and its dissociation occur in very short time $\left(t_{1 / 2}<1 \mathrm{sec}\right)$ [14], and such processes are generally expected to be much faster than many physiological processes [15]. CDs have significant influence on drug dissolution, bioavailability, safety, and stability, and therefore, they are increasingly used as excipients in drug formulations [16]. CDs are also widely used in the pharmaceutical applications because of their ready availability and cavity size suitable for the widest variety of drugs. However, the low aqueous solubility and nephrotoxicity limited the use of $\beta$-CD especially in parenteral drug delivery. Chemically modified CD or their derivatives have been prepared to extend the physicochemical properties (such as enhanced aqueous solubility, physical and microbiological stability, and reduced parenteral toxicity) and inclusion capacity of parent CDs $[17,18]$.

The hydroxypropyl- $\beta$-cyclodextrin (HP- $\beta$-CD) shows the best solubility $(500 \mathrm{mg} / \mathrm{mL})$ and lowest toxicity [19].
From the pharmaceutical point of view, the HP- $\beta$-CD presents the following advantages: increases the active substance solubility, bioavailability, and dissolution rate; reduces the side effects; and contributes to the stabilization of the pharmaceutical formulations [20]. Inclusion complexes of HP- $\beta$-CD are of great interest in pharmaceutical application, and they have been used to increase the solubility of a great number of various drugs [16, 21-26]. Rubim et al. [27] followed the effect of the CD type as $\beta$-cyclodextrin, methyl- $\beta$-cyclodextrin, and 2-hydroxypropyl- $\beta$-cyclodextrin on the complexation with AMD and release ability. The enhanced solubility and dissolution may help to improve in vivo performance. Only a few studies are reported on the formation and characterization of inclusion complexes of HP- $\beta$-CD with amiodarone [27], but no detailed studies on loading and release of the drug have been found, and there are no pharmacokinetic data available about these systems.

In our previous papers, it was established that the dissolution rate of amiodarone from the inclusion complex was considerably increased as compared to dissolution of the pure drug. It has been established that the complexation of amiodarone with $\mathrm{HP}-\beta-\mathrm{CD}$ offers the possibility to increase its water solubility without the modification of its original structure [28-31]. It is well known that the type of $\beta-C D$ can influence the formation as well as the performance of $\operatorname{drug} / \beta$-CD complexes $[32,33]$.

In the present study, the effect of the substitution degree and molecular weight of the 2-hydroxypropyl- $\beta$ cyclodextrin on the inclusion complex formation with the amiodarone and their physicochemical properties have been followed; also, the ability of loading and controlled release was studied by in vitro studies. The objective of this research was to evaluate the AMD bioavailability from the inclusion complexes as a formulation for oral administration with modified release and with optimal biopharmaceutical properties. Therefore, the second part of the paper reports some pharmacotechnical studies, where the inclusion complex with the best solubility was included in matrix tablets in order to obtain a modified-release behavior. In this respect, two formulations of matrix tablets were developed: one containing the inclusion complex with the optimal solubility and one containing only pure AMD as a control sample. The kinetics of AMD release from the two formulations was evaluated by in vitro and in vivo studies.

The disadvantage of oral administration is the extremely slow absorption of AMD from the oral forms and also low and variable bioavailability. The optimization of the pharmacokinetic properties was achieved by incorporation of the inclusion complexes in matrix tablets containing hydrophilic polymers such as Kollidon ${ }^{\circledR}$ SR (KOL) and chitosan (CHT) as excipients; finally, therapeutic systems with controlled prolonged release for oral delivery have been obtained.

The solubility enhancement of AMD by the inclusion in complexes with HP- $\beta$-CD was of 4-22 times. Depending on $\mathrm{HP}-\beta-\mathrm{CD}$ amount in the complex composition at a faster dissolution rate with small difference concerning the HP- $\beta$ $\mathrm{CD}$ type was found. The formulations of $\mathrm{AMD} / \mathrm{HP}-\beta-\mathrm{CD}$ inclusion complexes both as powdered form and matrix tablets showed superior pharmacokinetic performance in 
TABLE 1: Characteristics of the HP- $\beta$-CD and codes of the samples.

\begin{tabular}{lccc}
\hline Compound & Abbreviation & Substitution degree (hydroxypropyl groups per glucose unit) & $\mathrm{Mw}(\mathrm{Da})$ \\
\hline 2-Hydroxypropyl- $\beta$-cyclodextrin A & HP- $\beta$-CD A & 0.6 & 1380 \\
2-Hydroxypropyl- $\beta$-cyclodextrin B & HP- $\beta$-CD B & 0.8 & 1460 \\
2-Hydroxypropyl- $\beta$-cyclodextrin C & HP- $\beta$-CD C & 1.0 & 1540 \\
\hline
\end{tabular}

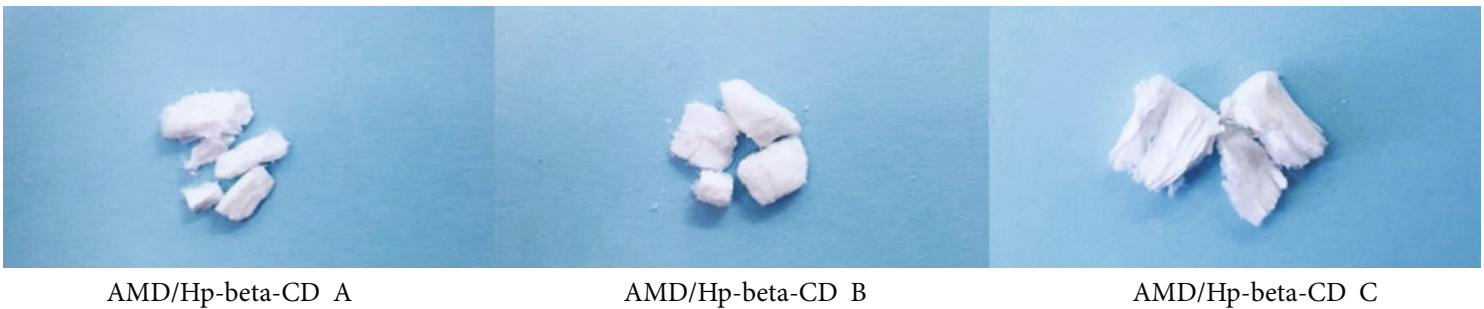

FIGURE 1: The aspects of the three amiodarone/2-hydroxypropyl- $\beta$-cyclodextrin complexes after freeze-drying.

improving loading and release properties of the insoluble AMD drug. An in vitro kinetic dissolution test reveals a complex mechanism occurring in three steps: the first one being attributed to a burst effect and the other two to different bonding existing in inclusion complexes. In vivo pharmacokinetic study from matrix tablets containing Kollidon ${ }^{\circledR}$ and chitosan also gave a multiple (at least two) peaks release diagram because of both structures of the inclusion complexes and also of different sites of absorption in biological media (digestive tract).

\section{Experimental}

2.1. Materials. $\mathrm{AMD}(\mathrm{Mw}=645.32 \mathrm{Da})$ of $99.85 \%$ purity was delivered by Zhejiang Sanmen Hengkang Pharmaceutical Co. Ltd., China. The three hydroxypropyl- $\beta$-cyclodextrins (HP- $\beta$ CDs) of $99.70 \%$ purity and with different substitution degrees and average molecular weights $(\mathrm{Mw})$ have been obtained from Roquette, France. Their characteristics are given in Table 1.

Polysorbate 80 with $99.50 \%$ purity was purchased from Sigma-Aldrich, Germany. It was used as a nonionic surfactant and emulsifier for AMD suspension.

Kollidon ${ }^{\circledR}$ SR (KOL) is a physical mixture constituted from $80 \%$ poly(vinyl acetate) with an average molecular weight $(\mathrm{Mw})$ of $450000 \mathrm{Da}$ and $20 \%$ polyvinylpyrrolidone (povidone) of $\mathrm{Mw}=40000 \mathrm{Da}$ [34]. KOL is a hydrophilic excipient used in formulations and preparation of the matrix tablets with modified-release dosage (delayed, prolonged, or targeted release) being both efficient and versatile [35].

Chitosan (CHT) with high molecular weight $\mathrm{Mw}=$ 190000-375000 Da and deacetylation degree $\geq 75$ of practical grade was purchased from BASF, Germany. It is a biodegradable and biocompatible polymer which acts as a promoter for the absorption at the gastrointestinal level of the hydrophobic substances [36, 37].

Avicel ${ }^{\circledR}$ PH: Microcrystalline Cellulose Avicel (Chemtrec, U.S.A., and Canada), Aerosil ${ }^{\circledR}$ : hydrophilic fumed silica with a specific surface area of $200 \mathrm{~m}^{2} \mathrm{~g}^{-1}$ (Degussa, Germany), and Magnesium Stearate (Union Derivan S.A., Spain) have been also used. All used compounds accomplish the quality requirements according with laws in forces. These excipients facilitate the application of the direct compression method, to obtain an optima AMD dispersibility in the powdered mixtures and association of hydrophilic (HP- $\beta-\mathrm{CD}, \mathrm{CHT})$ and hydrophobic substances (AMD).

\subsection{Methods}

2.2.1. Preparation of the Inclusion Complexes. To obtain the inclusion complexes of the bioactive compound AMD and the three HP- $\beta$-CDs, a $0.179 \mathrm{~g}$ amount of HP- $\beta$-CD was dissolved in $5 \mathrm{~mL}$ of water, and then, AMD was added in a molar ratio of $1: 1$. The mixtures have been stirred for 3 days at room temperature, and finally, three opaque solutions have been obtained, the inclusion complexes being partially soluble. The free noncomplexed AMD is separated by centrifugation at $600 \mathrm{rpm}$ for $10 \mathrm{~min}$. at room temperature. Then, the balloon flasks containing solution mixtures have been frozen with liquid nitrogen and dried by lyophilization for $24 \mathrm{~h}$ by means of a Labconco FreeZone 2.5 (Kansas City, Mo, SUA) system, and they have been kept in a desiccator. The AMD/HP- $\beta$-CD A, AMD/HP- $\beta$-CD B, and AMD/HP- $\beta$-CD $\mathrm{C}$ complexes have been obtained with the three corresponding HP- $\beta$-CDs. In the dry state, all samples look like homogeneous white masses with flake-like structures, the compactness decreasing from complex A to complex $\mathrm{C}$ (Figure 1).

\subsubsection{Characterization of the Inclusion Complexes}

(1) Scanning Electronic Microscopy (SEM) Examination. Scanning electronic microscopy (SEM) examination was carried out by using a Quanta 200 scanning electronic microscope (FEI Company, Hillsboro, OR, USA), with an integrated EDX system, and GENESIS XM 2i EDAX (FEI Company, Hillsboro, OR, USA) and with a SUTW detector, at an accelerating voltage of $20 \mathrm{kV}$ and without any further treatments, at different magnifications given on micrographs.

(2) Dynamic Light Scattering (DLS) and Zeta Potential Measurements. The particle size and distribution of the HP$\beta$-CDs and HP- $\beta$-CD/AMD complexes were analyzed by the dynamic light scattering (DLS) method using a Zetasizer 
Nano ZS apparatus (Malvern Instruments, Enigma Business Park, UK) equipped with a He-Ne laser $(\lambda=633 \mathrm{~nm})$ and operated at a scattering angle of $173^{\circ}$. The system uses a noninvasive back scatter (NIBS) technology (which reduces the multiple scattering effects) wherein the optic is not in contact with the sample. During determinations, the Mie method is applied over the whole measuring range from $0.6 \mathrm{~nm}$ to $6 \mu \mathrm{m}$.

The apparent hydrodynamic diameter $\left(D_{\mathrm{H}}\right)$ of the analyzed samples was determined using the Stokes-Einstein relation:

$$
D_{\mathrm{H}}=\frac{k T}{3 \pi \eta D},
$$

where $D_{\mathrm{H}}$ is the hydrodynamic diameter, $k$ is the Boltzmann constant, $T$ is the absolute temperature of $298.15 \mathrm{~K}, \eta$ is the viscosity, and $D$ is the diffusion coefficient.

Zeta potential $(\zeta)$ was determined on the same Zetasizer Nano ZS device by using the Smoluchowski relationship:

$$
\zeta=\frac{\eta \mu}{\varepsilon},
$$

$k \alpha>>1$, where $\mu$ is the electrophoretic mobility, $\eta$ is viscosity, $\varepsilon$ is dielectric constant, and $k$ and $\alpha$ are the Debye-Hückel parameter and particle radius, respectively.

From each sample dispersed in ultrapure water at a concentration of $0.06 \mathrm{~g} \mathrm{dL}^{-1}$, a good dispersion was obtained by simple dissolution, and to improve it, the ultrasonication was applied for $2 \mathrm{~h}$, and then, the samples were analyzed in triplicate at a constant temperature $\left(25^{\circ} \mathrm{C}\right)$ after $2 \mathrm{~min}$ of equilibration.

(3) ATR-FTIR Spectroscopy. The ATR-FTIR spectra have been recorded at $2 \mathrm{~cm}^{-1}$ resolution with 64 scans by means of a Bruker VERTEX 70 spectrometer (Bruker, Ettlingen, Germany), in the absorbance mode, by the Attenuated Total Reflection Fourier-Transform Infrared Spectroscopy (ATRFTIR) technique using a Golden Gate system equipped with diamond crystal with an incidence angle of $45^{\circ}$. Penetration thickness was about 100 microns. Background and sample spectra were recorded in the 600 to $4000 \mathrm{~cm}^{-1}$ wavenumber range with a resolution of $2 \mathrm{~cm}^{-1}$. For each sample, the evaluations were made on the average spectrum obtained from three recordings. The processing of spectra was achieved using ORIGIN programs.

(4) ${ }^{1} H$-NMR Spectroscopy. The ${ }^{1} \mathrm{H}-\mathrm{NMR}$ spectra and twodimensional ${ }^{1} \mathrm{H}-{ }^{1} \mathrm{H}$ chemical shift correlation spectra (COSY) were acquired on a Bruker NEO-1 $400 \mathrm{MHz}$ spectrometer (Bruker, Germany) equipped with a $5 \mathrm{~mm}$ QNP direct detection probe and z-gradients. For the NMR analysis, the compounds (HP- $\beta-\mathrm{CD}$ and complexes) were dissolved in deuterated dimethyl sulfoxide, DMSO-d6, while the amiodarone drug was dissolved in deuterated chloroform, $\mathrm{CDCl}_{3}-\mathrm{d}$, because it is not soluble in DMSO-d6. Deuterated solvents have been purchased from VWR Chemicals, and they have a purity of $99.80 \% \mathrm{D}$.
(5) UV-Visible Spectroscopy and Phase Solubility Studies. The direct titration method was performed by using an UVVIS spectrophotometric method (Cary 60 UV-VIS spectrophotometer; Agilent Technologies) at room temperature. One component of the complex (generally the HP- $\beta-\mathrm{CD}$ ) is gradually added at a fixed concentration of the other component of the system (the guest-AMD). Thus, for all experiments, a $0.018 \times 10^{-3} \mathrm{M}$ aqueous solution of AMD was prepared. While the AMD guest was kept at a constant concentration, the HP- $\beta$-CD host concentration varied from 0.4 to $8 \times 10^{-3} \mathrm{M}$. Each solution was kept under agitation for 12 hours. The UV absorption spectrum of each sample was recorded with a $1 \mathrm{~mm}$ thick quartz cuvette, and the variation in the absorbance peak of the AMD guest was monitored at $242 \mathrm{~nm}$. Because HP- $\beta$ CDs are silent (they do not absorb), the analysis complexity is reduced.

The phase solubility method is widely used to study inclusion complexation. It examines the effect of a solubilizer such as CD or ligand, on the drug which should be solubilized. The phase solubility tests were carried out according to the method described by Higuchi and Connors [38]. Briefly, an excess amount of AMD was added to $10 \mathrm{~mL}$ HP$\beta$-CDs in aqueous solutions at concentrations ranging from 0.2 to $10 \mathrm{M}^{-3}$. The flasks were covered to avoid solvent loss and then stirred for $24 \mathrm{~h}$ at room temperature. The resulting dispersions of $\mathrm{AMD} / \mathrm{HP}-\beta-\mathrm{CD}$ were filtered, and the solution concentration was determined by an UV-VIS spectrophotometric method, the recording being made at $242 \mathrm{~nm}$ wavelength characteristic to AMD for which a calibration curve was previously drawn. All the experiments were performed in triplicate. The apparent stability constants $\left(K_{\mathrm{c}}\right)$ of the complexes were calculated in accordance with Equation (3) from the slope of phase solubility diagrams, where the intercept $\left(S_{0}\right)$ is the intrinsic solubility of AMD in water, in the absence of cyclodextrins:

$$
K_{\mathrm{c}}=\frac{\text { slope }}{S_{0}(1-\text { slope })} .
$$

Gibbs free energy of complexation was evaluated using the well-known relation:

$$
\Delta \mathrm{G}=-\mathrm{RT} \ln \mathrm{K}_{\mathrm{c}}
$$

where $R$ is the gas constant $\left(8.314 \mathrm{~J} \mathrm{~mol}^{-1} \mathrm{~K}^{-1}\right)$ and $T$ is absolute temperature in Kelvin $(298.15 \mathrm{~K})$.

(6) Differential Scanning Calorimetry (DSC). Differential scanning calorimetry (DSC) was performed for thermal characterization of the samples, using a DSC 823e from Mettler Toledo instrument (Columbus, Ohio, USA) calibrated with indium as the standard. The samples weighing between 2 and $3.5 \mathrm{mg}$ were packed in aluminum pans and placed in the DSC cell. They were heated from ambient temperature to $180^{\circ} \mathrm{C}$ at a heating rate of $10^{\circ} \mathrm{C} \mathrm{min}^{-1}$. Melting temperature $\left(T_{m}\right)$, melting enthalpy 
$(\Delta \mathrm{Hm})$, and degradation temperature $\left(T_{d}\right)$ were obtained from first heating run.

(7) Loading Degree of the $A M D$ into $H P-\beta-C D / A M D$ Inclusion Complexes. The quantitative determination of the loading degree was realized by the HPLC method. In order to determine the loading capacity of the complexes with AMD, the $30 \mathrm{mg}$ complex samples were used. The 3.0-5.0 mg inclusion complex was dissolved in $10 \mathrm{~mL}$ methanol, and then, $1.0 \mathrm{~mL}$ from the obtained solution was diluted to $10 \mathrm{~mL}$ mobile phase resulting in $0.0155 \mathrm{mg}$ AMD $\mathrm{mL}^{-1}$. The free (noncomplexed) AMD is separated by centrifugation at $600 \mathrm{rpm}$ for $10 \mathrm{~min}$ at room temperature. The supernatant is collected and analyzed by the HPLC method. The reference sample was methanolic solution of Cordarone (AMD. $\mathrm{HCl}$ commercial product) $\left(c=0.05 \mathrm{mg} \mathrm{mL}^{-1}\right)$.

HPLC was performed by means of a chromatograph of a Thermo Fisher Surveyor type (Thermo Fisher, San Jose, USA) equipped with a UV-VIS detector with multiple Diode Array Detectors and a Thermo Fisher-Hypersil Betasil C18 $150 \mathrm{~mm} \times 4.6 \mathrm{~mm}$ column (Thermo Fisher, San Jose, USA); the particle size dimension was of $5 \mu \mathrm{m}$. The column temperature was kept constant at $45 \pm 0.2^{\circ} \mathrm{C}$. As the mobile phase, a mixture of formic acid $0.5 \%$ and methanol in the 25/75 $\mathrm{v} / \mathrm{v}$ ratio was used at a flow rate of $0.7 \mathrm{~mL} \mathrm{~min}^{-1}$. The injection volume for each determination was $20 \mu \mathrm{L}$. AMD was detected by UV spectrum at its characteristic wavelength at $254 \mathrm{~nm}$. The recorded retention time was $4.51 \mathrm{~min}$. The procedure is described in detail in our previous paper [39].

(8) In Vitro Dissolution Tests of AMD from Inclusion Complexes. The AMD dissolution profiles have been studied into two dissolution media with different $\mathrm{pHs}$, namely, $\mathrm{HCl}$ $0.1 \mathrm{~N}$ solution with $\mathrm{pH}=1.2$ (simulating media for gastric fluids) and phosphate buffer solution with $\mathrm{pH}=6.8$ (simulating media for intestinal fluids). The experiments were carried out by means of a dissolution test station type II, Hanson SR 8 Plus Series (Hanson Research Co., Chatsworth, USA) provided with two blades. The experiments have been performed according to the requirements described in Romanian [40], European [41], and United States Pharmacopoeia (USP) [42] with specifications for liquid and solid pharmaceutical formulations. The following protocol was followed: $100 \mathrm{mg}$ of pure AMD quantity and equivalent amount of complexes which contain also $100 \mathrm{mg}$ AMD evaluated according to loading degree were added into $500 \mathrm{~mL}$ of each above-mentioned dissolution medium. Temperature was kept constant at $37 \pm 0.5^{\circ} \mathrm{C}$, and the stirring speed was of $60 \mathrm{rpm}$. Aliquots $(2 \mathrm{~mL})$ of the dissolution medium were withdrawn, examined, and replaced with fresh dissolution media at predetermined time intervals of 5 minutes in the first 30 minutes, then at intervals of 10 minutes for the next $2 \mathrm{~h}$ and then at intervals of 30 minutes for the next $2 \mathrm{~h}$. The AMD in prevailed solution was quantitatively determined by the above-described and validated HPLC method [39]. Tests have been made in triplicate, and the average value was given.
TABLE 2: Matrix tablet formulations containing AMD and/or HP- $\beta$ CD/AMD B complex and auxiliary excipients.

\begin{tabular}{lcc}
\hline \multirow{2}{*}{ Substance } & \multicolumn{2}{c}{ Formulation composition (mg/tablet) } \\
& Formulation F & Formulation Fc \\
\hline AMD & 200 & - \\
AMD/HP- $\beta$-CD B & - & 200 \\
KOL & 240 & 240 \\
CHT & 18 & 18 \\
Aerosil & 6 & 6 \\
Stearate & 3 & 3 \\
Avicel & \multicolumn{3}{c}{ Up to $600 \mathrm{mg}$} \\
\hline
\end{tabular}

(9) Kinetic Release of AMD from Inclusion Complexes. The release kinetic parameters were calculated using the equation proposed by Korsmeyer et al. [43]:

$$
\frac{M_{t}}{M_{\infty}}=k_{r} t^{n_{r}}
$$

where $M_{t} / M_{\infty}$ represents the fraction of the drug released at time $t, k_{r}$ is a constant incorporating characteristics of the macromolecular matrix, and $n_{r}$ is the diffusion exponent, which is indicative for the release mechanism. In the above equations, a value of $n_{r}=0.5$ or smaller indicates a Fickian diffusion mechanism of the drug from the matrix - as for step 1 at both $\mathrm{pHs}$ and step 2 at $\mathrm{pH} 6.8$; a value $0.5<n_{r}<1$ indicates an anomalous or non-Fickian behavior. This is characteristic to step 1 and step 2 at $\mathrm{pH} 6.8$. When $n_{r}=1$, a case II transport mechanism is involved while $n_{r}>1$ indicates a special case II transport mechanism of step 3 at pH 1.2 (see below).

2.2.3. Matrix Tablet Formulation Preparation. Two formulations based on KOL and $\mathrm{CHT}$ as matrix constituents have been prepared, one containing only pure AMD as the active compound (designed as F) and another containing AMD/ $\mathrm{HP}-\beta$-CD B complex (designed as $\mathrm{Fc}$ ). The AMD/HP- $\beta$ CD B inclusion complex was selected for the oral therapeutic system preparation because of its good physicochemical and pharmacotechnical properties in comparison with the other two AMD/HP- $\beta$-CD A and AMD/HP- $\beta$-CD C complexes as it was demonstrated by their characterization (see below). The purpose of this strategy was to evaluate the influence of the therapeutic system type on the oral biodisponibility of AMD and to analyze the influence of AMD complexation with HP- $\beta-C D$ B on this pharmacokinetic parameter. In Table 2 are presented the compositions of the two oral formulations, where $\mathrm{KOL}$ and $\mathrm{CHT}$ and other specific auxiliary excipients were included $[37,44,45]$.

An Erweka AR 403 device (Erweka GmbH, Heusenstamm, Germany) was used for these preparations in the following conditions: rotation speed $400 \mathrm{rpm}$ for $5 \mathrm{~min}$; after that, the mixtures were sieved with an electromagnetic sieve EM-8 (Erweka GmbH, Heusenstamm, Germany). Direct compression of the mixtures was done with a Korsch EK0 single-punch station press (Korsch AG, Berlin, Germany) (punch diameter $9 \mathrm{~mm}$, compression force $8-10 \mathrm{kN}$ ). The 
matrix tablets with an average diameter of $12 \mathrm{~mm}$ and thickness of $4.6 \mathrm{~mm}$ have been obtained (Table 2).

(1) Pharmacotechnical Characterization of the Matrix Tablets Containing $A M D$ and $A M D / H P-\beta-C D B$. The evaluation of the pharmacotechnical characteristics (diameter, thickness, and average mass) of the tablets was performed according to the $10^{\text {th }}$ Romanian Pharmacopoeia and $8^{\text {th }}$ European Pharmacopoeia [40, 41]. Weighing was made by means of an electronic balance Radwag WPE 60, on 20 tablets for mass and dose uniformity determination. Mechanical resistance was performed on 10 tablets on a Schleuniger Pharmatron tablet hardness tester 8M (Sotax AG, Aesch, Switzerland) and the friability on 20 tablets on Schleuniger Pharmatron FT II friability tester (Sotax AG, Aesch, Switzerland), at $100 \mathrm{rpm}$ for $4 \mathrm{~min}$. All pharmacotechnical characteristics have been performed comparatively for the two studied formulations, F and Fc. The dose uniformity was established using a validated HPLC procedure through the quantitative determination of AMD from a fine powder obtained by the trituration and the homogenization of 20 tablets with known average masses. The SD should be of $\pm 5 \%$ [40] in all cases. According to pharmacotechnical specifications for the preparation of modified-release tablets, the release profiles of the active substance from such type of tablets must be analyzed by determining the difference factor $\mathrm{f} 1$ and the similarity factor $\mathrm{f} 2$ between two or more formulations [46].

Hydration capacity or swelling degree was determined by using a dissolution test station type II, Hanson SR 8 Plus Series (Hanson Research Co., Chatsworth, USA). Matrix tablets have been introduced in $1000 \mathrm{~mL}$ distilled water at $37^{\circ} \mathrm{C}$ at $60 \mathrm{rpm}$. At the predetermined time intervals $(1 \mathrm{~h})$, samples were prevailed from hydration medium; the water excess on the surface was removed by wiping with filter paper, and then, they have been weighed. The swelling degree was evaluated using relation:

$$
\mathrm{SD}=\frac{W_{\mathrm{t}}-W_{\mathrm{o}}}{W_{\mathrm{o}}} 100
$$

where SD is the swelling degree, $W_{t}$ is mass of the sample at time $t$, and $W_{\mathrm{o}}$ is dry mass of the tablet.

(2) In Vitro Release Tests of AMD from Matrix Tablets. In vitro release of AMD from the matrix tablets $\mathrm{F}$ and $\mathrm{Fc}$ have been determined into simulating gastric fluid with $\mathrm{pH}=1.2$ and into simulating intestinal fluid with $\mathrm{pH}=6.8$ until the release amount reached equilibrium (maximum of 10 hours). The method uses the same equipment and working conditions as described above at In Vitro Dissolution Tests of AMD from Inclusion Complexes. Tests have been made in triplicate, and the average value was given.

(3) In Vivo Single-Dose Pharmacokinetic Study. The singledose pharmacokinetics of the new formulation Fc, which contains AMD/HP- $\beta$-CD B complex, was investigated comparatively with formulation F1 containing pure AMD.
Ethics Statement. The experiment was approved by the Ethics Committee of "Grigore T. Popa" University of Medicine and Pharmacy of Iasi, Romania (No. 23983/2014) and was carried out in accordance with the European regulations concerning the studies with animals. The study was carried out on male Wistar rats (average weight of 250$300 \mathrm{~g}$ ) provided by the Cantacuzino Institute-Bucharest, Romania.

Experimental Design and Sampling. The rats were housed in polypropylene cages with controlled environmental conditions of temperature $\left(21 \pm 2^{\circ} \mathrm{C}\right)$ and humidity (50-70\%) and successive light and dark of $12 \mathrm{~h}$ cycles and on ad libitum access to food and water. After three days of acclimation, on the evening of the day before administration of the substances and until $4 \mathrm{~h}$ post administration, no food was administered to all rats, but they had free access to drinking water. A temporary cannula with a $24 \mathrm{G}$ catheter was placed in the lateral tail vein for blood collection as "free flowing," after warming the animals at $37^{\circ} \mathrm{C}$ for 10 minutes in order to allow tail vein vasodilation. The substances were administered by oral gavage as freshly prepared solutions, as a single oral dose of $100 \mathrm{mg}$ substance/kg body in a volume of $0.2 \mathrm{~mL}$ $(100 \mathrm{~g})^{-1}$ body. Each animal was weighed before the administration of the substances. Rats were randomly assigned into three groups of six animals each, according to the following protocol: Group 1 (control AMD group) received free drug suspension of AMD in aqueous polysorbate 80 $(c=0.1 \% w / v)$, Group 2 (F group) received F formulation, and Group 3 (Fc group) received Fc formulation. Approximately $0.3 \mathrm{~mL}$ of blood was collected into heparinized tubes at 0 (predose), $0.25,0.5,1,2,3,4,5,6,8,10,12$, 24 , and $48 \mathrm{~h}$ postdose. A heparin flush of $0.1 \mathrm{~mL}$ was used after placement and between blood samples to prevent clotting. Blood samples were centrifuged at $4000 \mathrm{rpm}$ for $10 \mathrm{~min}$; then, plasma was stored at $-20^{\circ} \mathrm{C}$ until analysis. Plasma samples were analyzed using the HPLC method. The lower limit of quantification for AMD was established at $5.83 \mu \mathrm{g} \mathrm{mL}^{-1}$.

Pharmacokinetics and Statistical Analysis. The plasma pharmacokinetic parameters directly determined by inspection of the individual drug plasma concentration versus time curves included the observed maximum plasma concentration $\left(C_{\max }\right)$ and the observed time $\left(t_{\max }\right)$ to reach maximum concentration. The area under the curve from $0 \mathrm{~h}$ to last determined experimental data $\left(\mathrm{AUC}_{0-t}\right)$ was estimated by the linear trapezoidal rule calculated from the individual AUCs from the plasma concentrations versus time curves. Noncompartmental pharmacokinetic analysis was performed using TopFit 2.0 Pharmacokinetic Software (Thomae $\mathrm{GmbH}$, Germany) to determine other main pharmacokinetic parameters: area under the plasma concentration time curve from time zero to infinity $\left(\mathrm{AUC}_{0-\infty}\right)$, elimination rate constant $\left(k_{\mathrm{e}}\right)$, and half-life $\left(t_{1 / 2}\right)$. The elimination rate constant $\left(k_{\mathrm{e}}\right)$ for each rat was estimated from the slope of the regression line of plasma concentrations versus time curves by the least squares regression analysis, and the apparent elimination half-life $\left(t_{1 / 2}\right)$ was calculated from the 


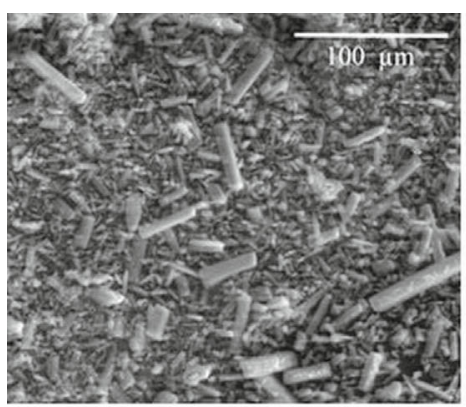

AMD

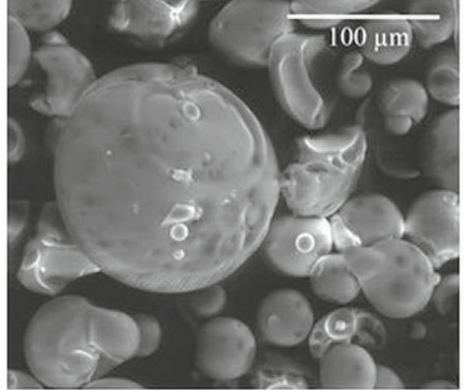

HP- $\beta$-CD A

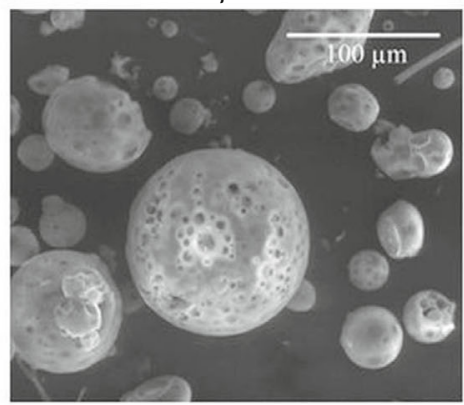

$\mathrm{HP}-\beta-\mathrm{CD} B$

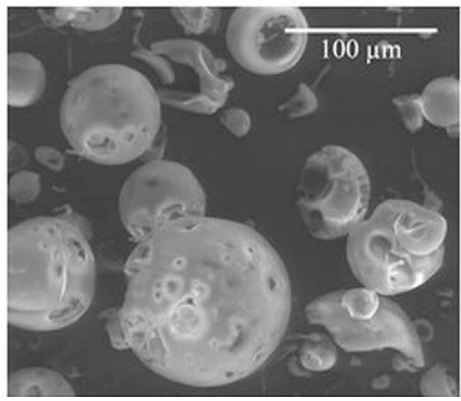

HP- $\beta$-CD C

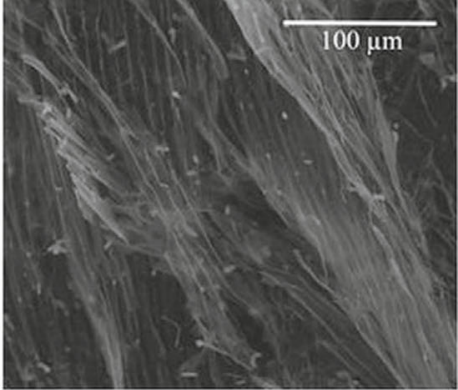

AMD/HP- $\beta$-CD A

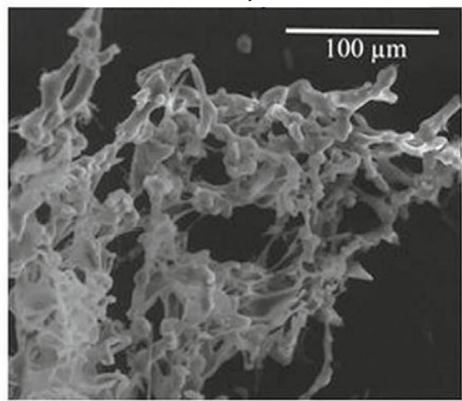

$\mathrm{AMD} / \mathrm{HP}-\beta-\mathrm{CD} \mathrm{B}$

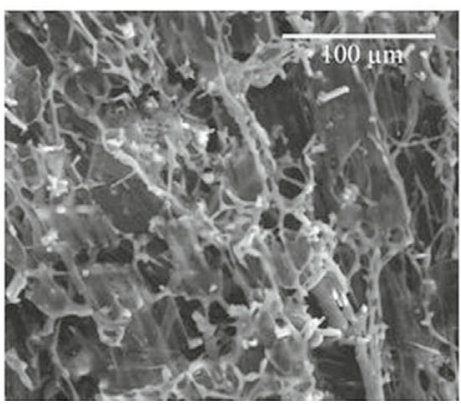

$\mathrm{AMD} / \mathrm{HP}-\beta-\mathrm{CD} \mathrm{C}$

FIGURE 2: SEM images of AMD, HP- $\beta$-CD, and corresponding inclusion complexes; magnification $100 \mu \mathrm{m}$ indicated on figure.

equation $t_{1 / 2}=\ln (2) / k_{\mathrm{e}}$. Systemic relative bioavailability $\left(F_{\mathrm{rel}}\right)$ was calculated by the following equation:

$$
F_{\text {rel }}=\frac{A U C_{\text {test }}}{A U C_{\text {reference }}},
$$

where $A U C_{\text {reference }}$ is the $A U C$ of free $A M D$ and $A U C_{\text {test }}$ is the AUC of the studied formulations. Results of pharmacokinetic studies were expressed as the mean \pm standard deviation (SD). Differences in the pharmacokinetic parameters were analyzed with the one-way analysis of variance (ANOVA) test using SigmaPlot 11 (SxST.it, Italy) software, and a $p$ value of $<0.05$ was considered significant.

\section{Results and Discussion}

3.1. Characterization of the 2-Hydroxypropyl-betacyclodextrins and Corresponding Inclusion Complexes

3.1.1. Scanning Electron Microscopy (SEM) Results. Given in Figure 2 are the SEM images of AMD, 2-hydroxypropylbeta-cyclodextrins, and corresponding inclusion complexes.
Powder of amiodarone exhibits cylindrical microcrystals of various sizes with tendency of aggregation. 2 -HP- $\beta$-CDs in the dry state look as spherical particles of various dimensions. The HP- $\beta$-CD A particles show a smooth surface, while the other two HP- $\beta$-CD B and HP- $\beta$-CD C show also spherical shapes, but on their surfaces, some porosities are evident, probably because of the most stable configuration at the high substitution degree. The morphology of the inclusion complexes is homogeneous, totally different in respect of those of the components (AMD and HP- $\beta$-CDs), and it is specific for each complex. Generally, they look as fibrous materials with a particular aspect and integrity for each of them depending on both substitution degree and molecular weight. The SEM image of the AMD/HP- $\beta$-CD B complex is very different in respect of those of other two complexes (AMD/HP- $\beta$-CD A and AMD/HP- $\beta$-CD C). This particular morphology and particle shape are indicative of the formation of the new solid phase.

The histograms in Figure 3 look as the curves with one peak for HP- $\beta$-CD A and HP- $\beta$-CD C samples. From these curves, the average diameters of the particles were determined at $20 \mu \mathrm{m}$ and $9 \mu \mathrm{m}$, respectively. The HP- $\beta$-CD B showed a bimodal histogram with two characteristic particle 


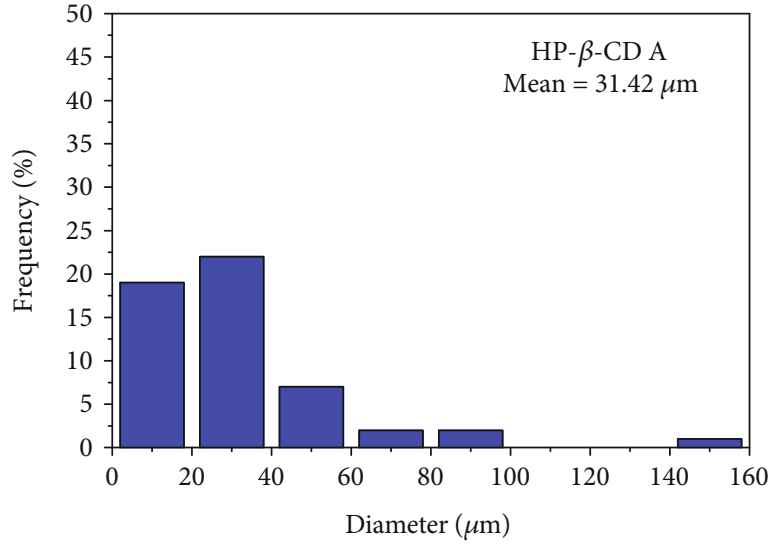

(a)

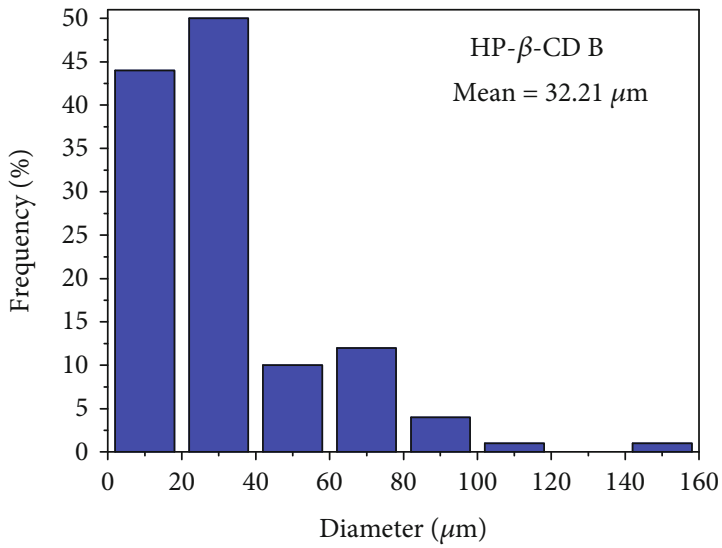

(b)

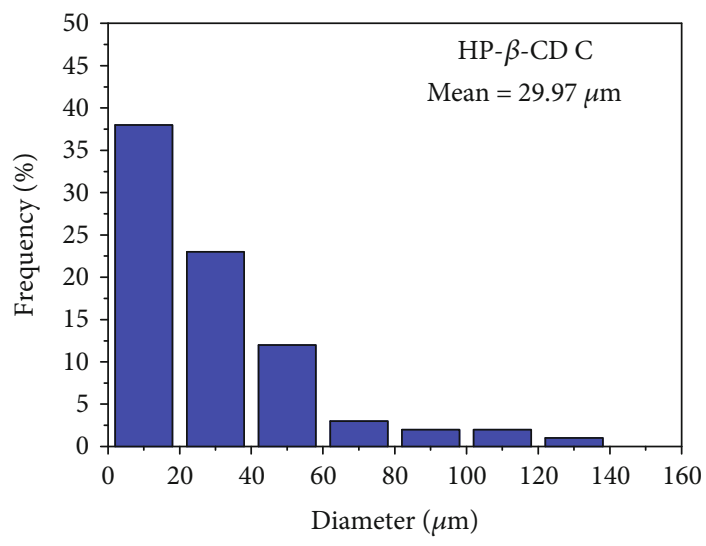

(c)

FIGURE 3: Size distributions of the particles in pure cyclodextrins: HP- $\beta$-CD A (a), HP- $\beta$-CD B (b), and HP- $\beta$-CD C (c). The mean diameter is given in legend.

diameters of $20 \mu \mathrm{m}$ and $70 \mu \mathrm{m}$; therefore, in this case, the particle distribution is particular.

\subsubsection{Dynamic Light Scattering (DLS) and Zeta Potential} Measurements. The DLS technique was employed to monitor the hydrodynamic size and colloidal stability of the HP- $\beta$ CDs and inclusion complexes dispersed in water. The obtained results are shown in Figure 4 and Table 3.

The size distribution profiles of HP- $\beta-\mathrm{CD}$ A and HP$\beta$-CD $\mathrm{C}$ resemble each other, showing one low-intensity peak at around $3 \mathrm{~nm}$ and a second main one which ranges from $140 \mathrm{~nm}$ to $165 \mathrm{~nm}$-Figures 4(a) and 4(b). Three different particle size populations coexist in the HP- $\beta$-CD B sample. The first one is the size population that can be observed as the first part of the curve with a diameter of $\sim 3 \mathrm{~nm}$ which is associated to the formation of small assemblies containing 2 or $3 \mathrm{HP}-\beta-\mathrm{CD}$ units [47]. A second size population appears at around $77 \mathrm{~nm}$ and a third one with the highest $D_{\mathrm{H}}$ at around $530 \mathrm{~nm}$. These results can be explained by the fact that $\mathrm{HP}-\beta$-CDs are known to self-assemble in aqueous solutions to form clusters with intermolecular linkage attributed to the $\mathrm{OH}$ groups located at the rims of the donut-shaped molecules [48]. Similar results have been obtained by Gonzalez-Gaitano et al. [49] who also used dynamic light scattering technology to study the association of CDs. They showed the formation of aggregates in aqueous solutions (with a $D_{\mathrm{H}}$ ranging from 124 to $172 \mathrm{~nm}$ ) together with nonassociated molecules.

The inclusion complexes displayed the different size distribution curves and histograms, being much homogeneous-Figures $4(\mathrm{a})$ and $4(\mathrm{~b})$. The $D_{\mathrm{H}}$ range from 189 to $295 \mathrm{~nm}$ and lower polydispersity index values when compared to those of the pure HP- $\beta$-CDs. The PDI is an indicator that measures the heterogeneity of the materials in aqueous solution, a smaller value indicating a narrower size distribution which is most likely related to a lesser tendency of the complexes to form aggregates.

The stability of the formed complexes can also be estimated by zeta potential assessment. The results (Table 3) demonstrate a stabilization of the complexes, AMD/HP- $\beta$ CD B showing the highest zeta potential value $(17.3 \mathrm{mV})$ which suggests that this is the most stable formulation, followed by AMD/HP- $\beta$-CD A $(15.7 \mathrm{mV})$ and AMD/HP- $\beta$ $\mathrm{CD} C(10.9 \mathrm{mV})$. The DLS results are in a good accordance with those obtained by SEM examination.

3.1.3. ATR-FTIR Results. The ATR-FTIR spectra of the three HP- $\beta$-CDs are similar (Figures $5(\mathrm{a})-5(\mathrm{c})$ ) and are in agreement with those reported in other papers [14, 50-55]. A characteristic absorption band of $\alpha$-type glycosidic bond was found 


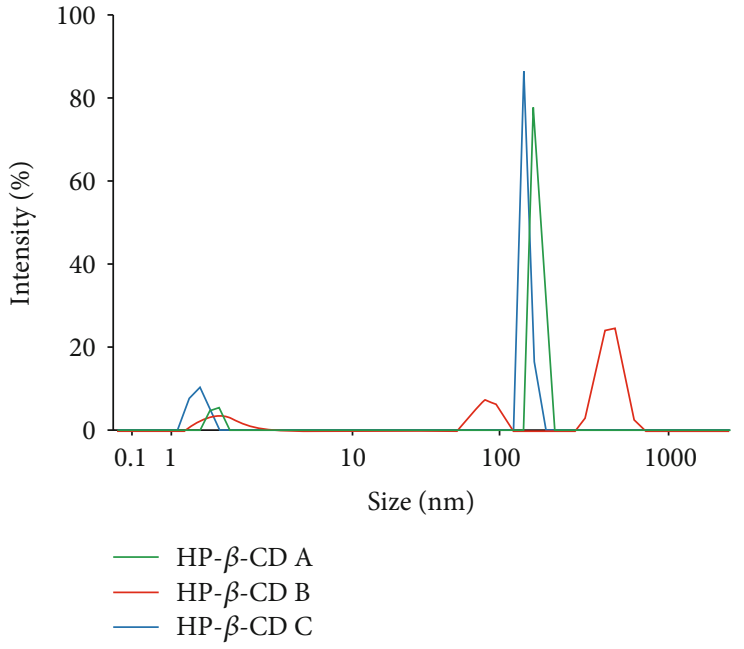

(a)

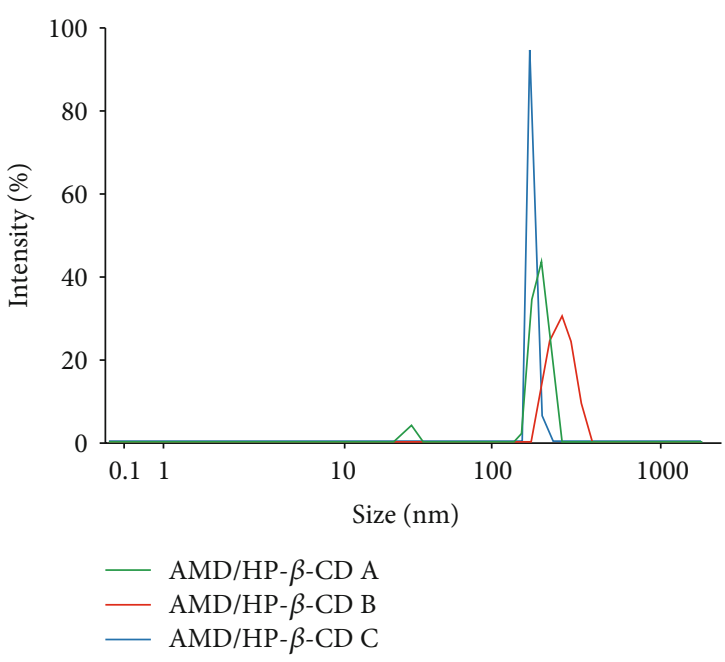

(b)

FIgURE 4: Size distribution curves of cyclodextrins and corresponding inclusion complexes: (a) of HP- $\beta$-CD A, HP- $\beta$-CD B, and HP- $\beta$-CD C and (b) of AMD/HP- $\beta$-CD A, AMD/HP- $\beta$-CD B, and AMD/HP- $\beta$-CD C in aqueous solution $\left(c=0.07 \mathrm{~g} \mathrm{dL}^{-1}\right)$ as determined by DLS.

TABLE 3: Z-average, PDI (polydispersity index), and zeta potential results.

\begin{tabular}{|c|c|c|c|c|c|c|}
\hline \multirow[t]{2}{*}{ Sample } & \multirow[t]{2}{*}{$Z$-average $(\mathrm{nm})$} & \multicolumn{3}{|c|}{ Size $(\mathrm{nm})$} & \multirow[t]{2}{*}{ PDI } & \multirow[t]{2}{*}{ Zeta potential $(\mathrm{mV})$} \\
\hline & & Peak 1 & Peak 2 & Peak 3 & & \\
\hline $\mathrm{HP}-\beta-\mathrm{CD} \mathrm{A}$ & 1174 & 3.7 & 164 & - & 0.945 & -0.222 \\
\hline $\mathrm{HP}-\beta-\mathrm{CD} \mathrm{B}$ & 1172 & 3.6 & 77 & 530 & 0.819 & -1.43 \\
\hline $\mathrm{HP}-\beta-\mathrm{CD} C$ & 2339 & 2.1 & 141 & - & 1.000 & -0.802 \\
\hline $\mathrm{AMD} / \mathrm{HP}-\beta-\mathrm{CD} \mathrm{A}$ & 882 & 33 & 221 & - & 0.685 & 15.7 \\
\hline $\mathrm{AMD} / \mathrm{HP}-\beta-\mathrm{CD} \mathrm{B}$ & 445 & - & 295 & - & 0.482 & 17.3 \\
\hline $\mathrm{AMD} / \mathrm{HP}-\beta-\mathrm{CD} \mathrm{C}$ & 1076 & - & 189 & - & 0.932 & 10.9 \\
\hline
\end{tabular}

at $850 \mathrm{~cm}^{-1}$, which indicates that HP- $\beta$-CDs were formed by glucopyranose units through $\alpha$-1,4-glycosidic bond [50].

The main bands corresponding to AMD are the following: 2964 and $2934 \mathrm{~cm}^{-1}$ assigned to a $\mathrm{CH}$ aliphatic stretching, $2457 \mathrm{~cm}^{-1}$ corresponding to tertiary amine stretch, and in the region of $1285 \mathrm{~cm}^{-1}$ the band referred to as ketone $\mathrm{C}=\mathrm{O}$ group bending [51]. The three complexes (AMD/HP- $\beta$-CDs $\mathrm{A}, \mathrm{B}$, and $\mathrm{C}$ ) show some differences in respect of spectra of their components (HP- $\beta$ $\mathrm{CD}$ and $\mathrm{AMD})$, which were evidenced in different spectral regions (Figure 5).

The $1022 \mathrm{~cm}^{-1}$ and $755 \mathrm{~cm}^{-1}$ bands from HP- $\beta$-CD had shifted to $1031 \mathrm{~cm}^{-1}$ and $750 \mathrm{~cm}^{-1}$ in case of the inclusion complexes formed with AMD. The $2457 \mathrm{~cm}^{-1}$ band in the AMD spectrum is much wide in the spectra of the inclusion complexes because of the combination of the stretching of the $\mathrm{C}-\mathrm{H}$ and $\mathrm{C}-\mathrm{C}$ bonds. The intensity of the group of bands placed between $1600 \mathrm{~cm}^{-1}$ and $950 \mathrm{~cm}^{-1}$ characteristic to the amiodarone decreases in the spectra of the inclusion complexes. The most evident decrease in intensity was observed in the case of the band from $1629 \mathrm{~cm}^{-1}$. Changes of the bands in spectra of the components of the inclusion complexes such as shifts and increase or decrease in intensity were reported by other authors [52] which were explained by the insertion of the benzene part ring into the electron-rich cavity of $\beta$ - cyclodextrin $[16,53]$ and due to the changes in the microenvironment which lead to the formation of hydrogen bonds and the presence of van der Waals forces during their interaction to form the inclusion complexes $[54,55]$.

As it can be seen in Figure 6(a) $\left(3600-2700 \mathrm{~cm}^{-1}\right.$ region), the bands in the spectrum of the inclusion complex $\mathrm{AMD} / \mathrm{HP}-\beta-\mathrm{CD} \mathrm{C}$ are much broader than those of the other two complexes, and also, some differences appear in the position of the $\mathrm{OH}$ and $\mathrm{CH}$ stretching, namely, $3373 \mathrm{~cm}^{-1}$ and $2924 \mathrm{~cm}^{-1}$.

The shapes of the spectra in the $1700-1200 \mathrm{~cm}^{-1}$ region are specific to each complex because of the variation in band position and in intensity, as observed in Figures 6(b) and 6(c).

Thus, differences between the three inclusion complexes of some characteristic band positions were found as complex A: $1657 \mathrm{~cm}^{-1}, 1563 \mathrm{~cm}^{-1}$, and $1555 \mathrm{~cm}^{-1}$; complex B: $1653 \mathrm{~cm}^{-1}$, $1558 \mathrm{~cm}^{-1}$; and complex C: $1655 \mathrm{~cm}^{-1}, 1646 \mathrm{~cm}^{-1}$ which can be assigned to $\mathrm{OH}$ bending and amide II. The band position is characteristic to each complex which means that also some interactions with substituent characterize these complexes due to the appearance of host-guest interactions.

3.1.4. ${ }^{1} H-N M R$ Results. NMR study is the most important tool which ascertains the inclusion phenomena of the guest 


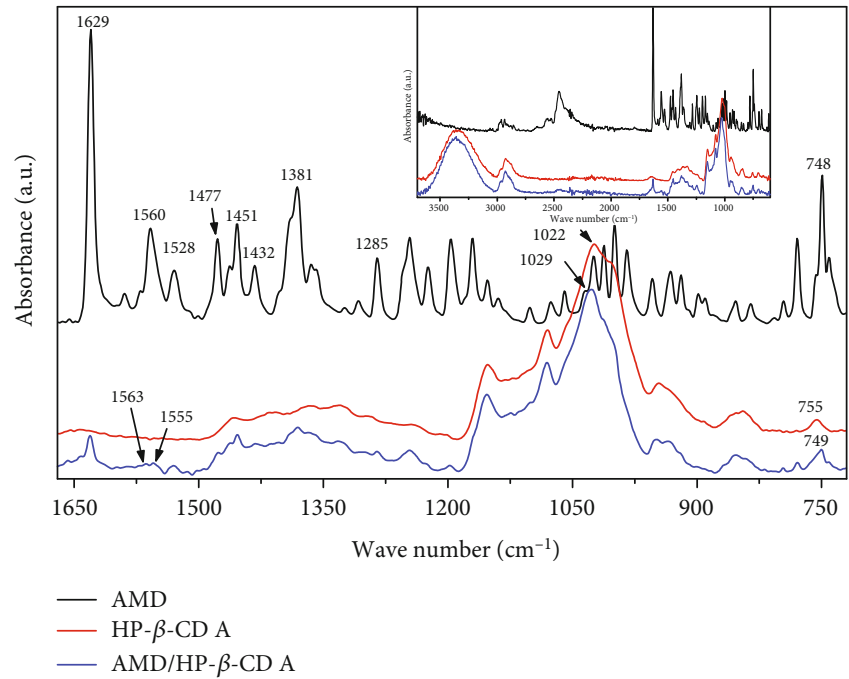

(a)

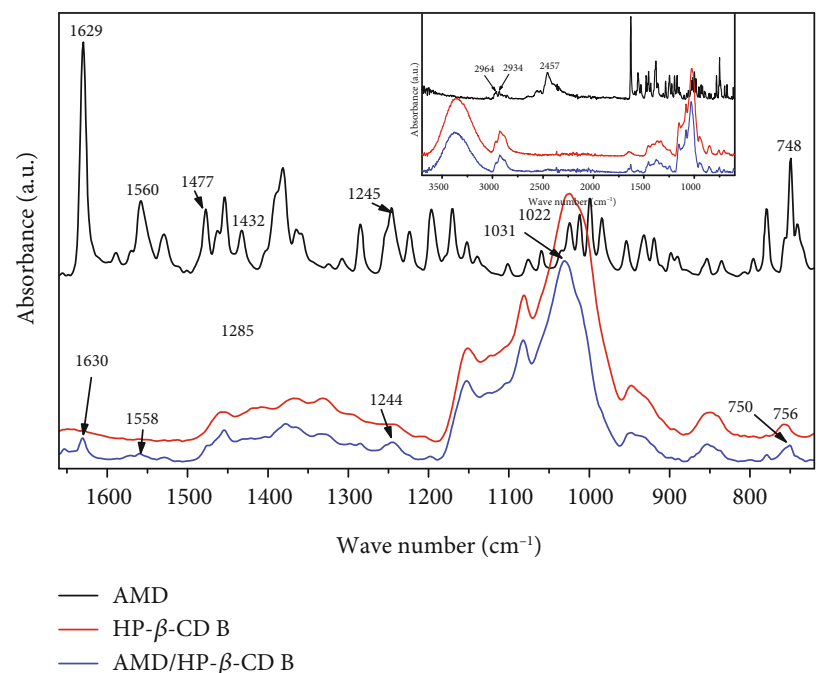

(b)

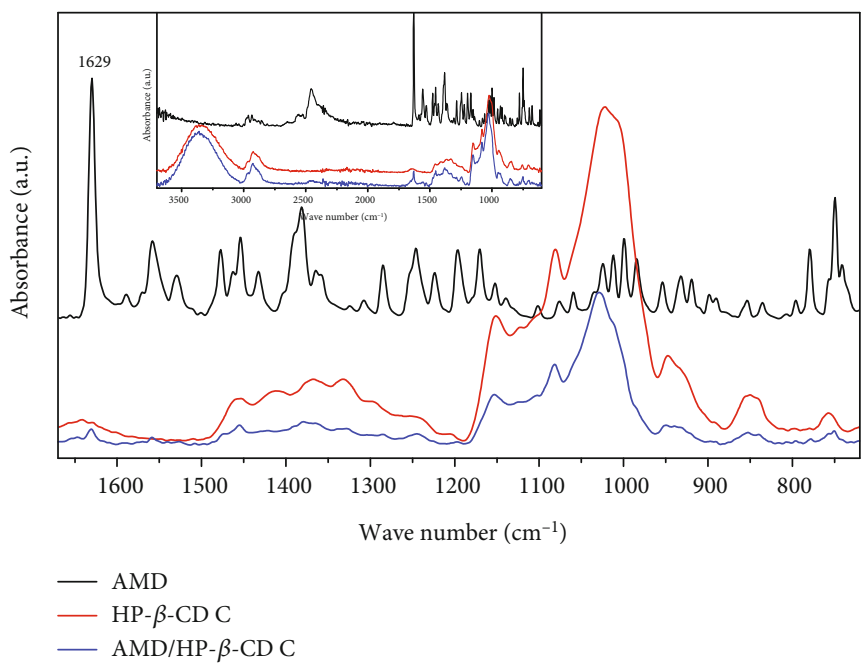

(c)

Figure 5: ATR-FTIR spectra of the inclusion complexes of AMD with the three 2 -HP- $\beta$-CDs: (a) AMD/HP- $\beta$-CD A, (b) AMD/HP- $\beta$-CD B, and (c) AMD/HP- $\beta$-CD C in respect of their components.

drug molecule inside the host CD molecule. The raw materials and the obtained complexes were characterized by ${ }^{1} \mathrm{H}-$ NMR-Figures 7(a) and 7(b).

Comparing the three superposed spectra, in the complex spectrum, the signals for the raw materials can be identified and the appearance of a new signal, as a singlet at 10.44 ppm-Figure 7.

In the 2D NMR spectrum (Figure 8), the couplings between HP- $\beta$-CD signals of 3.38 and 3.68 ppm and new signal at $10.44 \mathrm{ppm}$ in the obtained complex can be observed. This signal indicates a direct $\mathrm{H}-\mathrm{H}$ connection between components of the complexes [56] and also suggested that the lipophilic aromatic ring of the AMD entered into the cavity of $\mathrm{HP}-\beta$-CD from the wider side [57].

3.1.5. UV-Visible Spectroscopy and Phase Solubility Study Results. The formation of inclusion complexes of AMD with the three types of HP- $\beta$-CDs in aqueous solutions was followed by the measurements of the absorbance at $242 \mathrm{~nm}$ which is characteristic to AMD as a function of $\mathrm{HP}-\beta-\mathrm{CD}$ concentration-Figure 9. The results show that the absorbance at $242 \mathrm{~nm}$ increases with the concentration of HP- $\beta$ $\mathrm{CD}$ which is due to the increase in AMD solubility (increased AMD solution concentration) as it is being included in the HP- $\beta$-CD cavity. It was clear that the solubility of $\mathrm{AMD} / \mathrm{HP}-\beta-\mathrm{CD}$ was much higher than that of pure AMD. These results indicated that $\mathrm{HP}-\beta-\mathrm{CD}$ formed inclusion complexes with AMD, which improved the aqueous solubility of AMD. It seems that the shapes of the UV-VIS spectra of the complex B are a little different from the other two; the main absorbance band $(242 \mathrm{~nm})$ is narrower, and values of maximum absorbance are smaller at each concentration.

The physicochemical properties of CDs, including their complexation ability, are affected by the type, number, and 


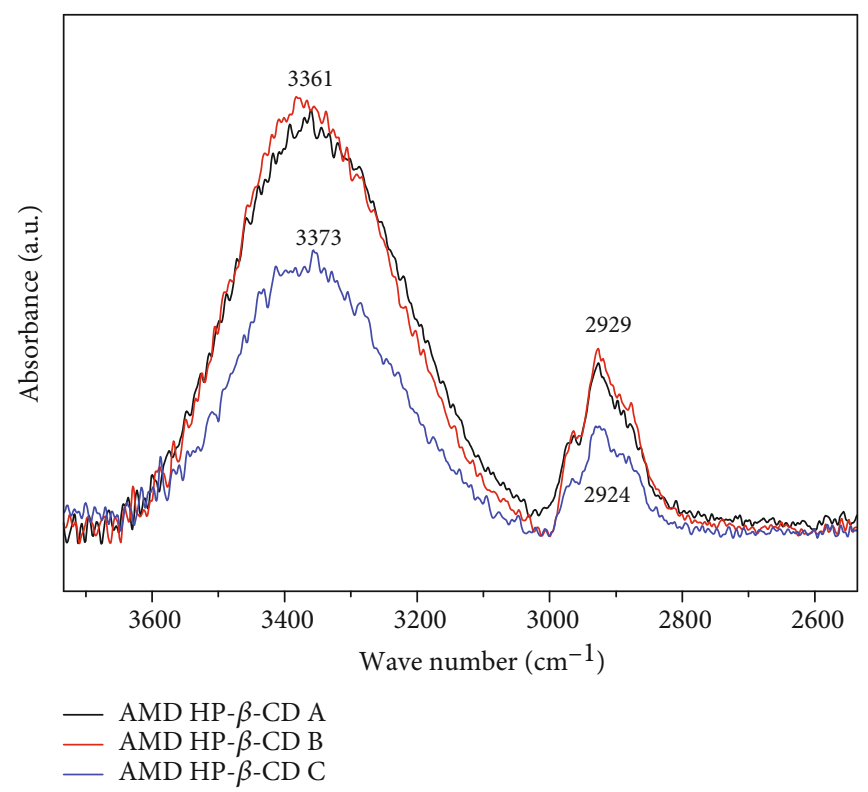

(a)

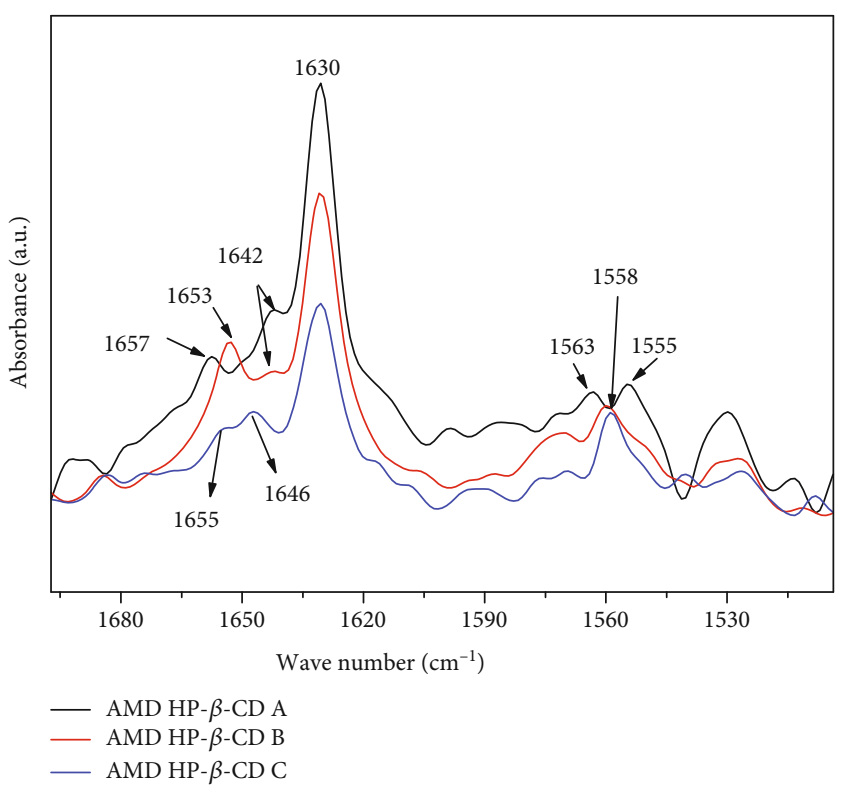

(b)

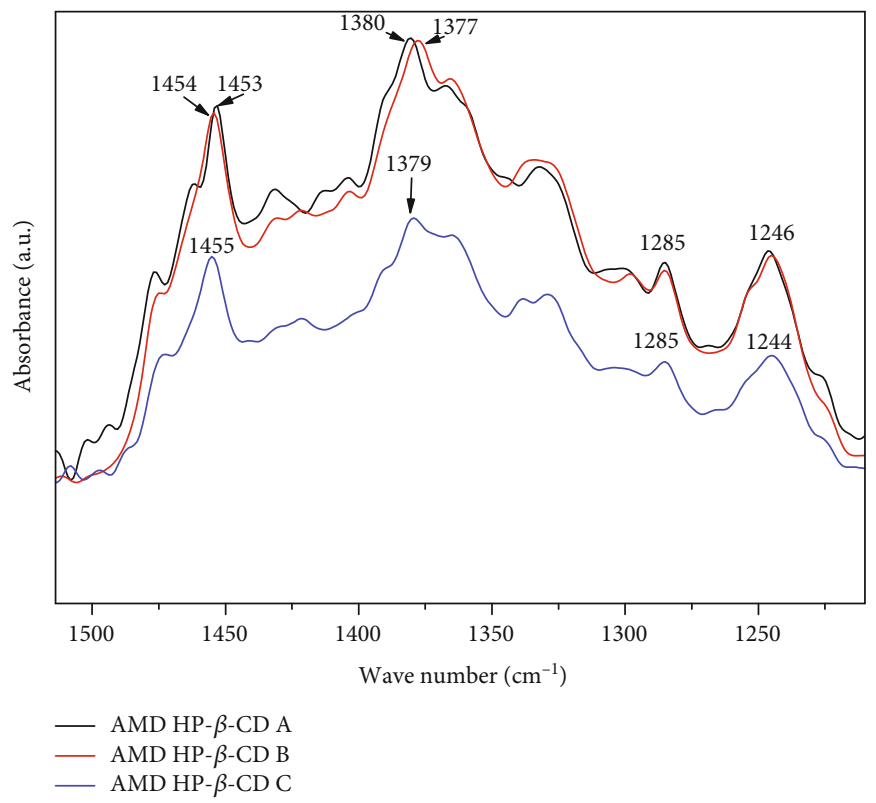

(c)

FIGURE 6: ATR-FTIR spectra of the three inclusion complexes in different spectral regions: (a) $2600-3600 \mathrm{~cm}^{-1}$, (b) $1530-1680 \mathrm{~cm}^{-1}$, and (c) $1200-1500 \mathrm{~cm}^{-1}$.

position of the substituents linked on the CD molecule [16]. The degree of substitution of the HP- $\beta$-CD, the substituent position, and purity also influence these properties. During the substitution reaction, new reactive sites are generated in HP- $\beta$-CD derivatives. Derivatives with a low degree of substitution showed the best complexation properties with low surface activities. As the substitution increased, the steric hindrances weakened the binding. All these effects are also dependent upon the particular guest, as the drug nature. In many cases, the effect of the degree of substitution differences in the binding capacity was small [58].
As observed in Figure 10, the AMD guest solubility linearly increased with increasing concentrations of HP$\beta$-CDs over the concentration range evaluated, indicating a 1:1 molecular complex formation between AMD and the three HP- $\beta$-CDs. For further experimental analyses, the molar ratio $(1: 1, \mathrm{AMD}: \mathrm{HP}-\beta-\mathrm{CD})$ was chosen to prepare the inclusion complexes as this ratio was also established in our previous study [28]. This is a phase $A_{L}$ type diagram which indicates the formation of water soluble inclusion complexes with linear increases of drug solubility as a function of HP- $\beta$-CD concentration. The 


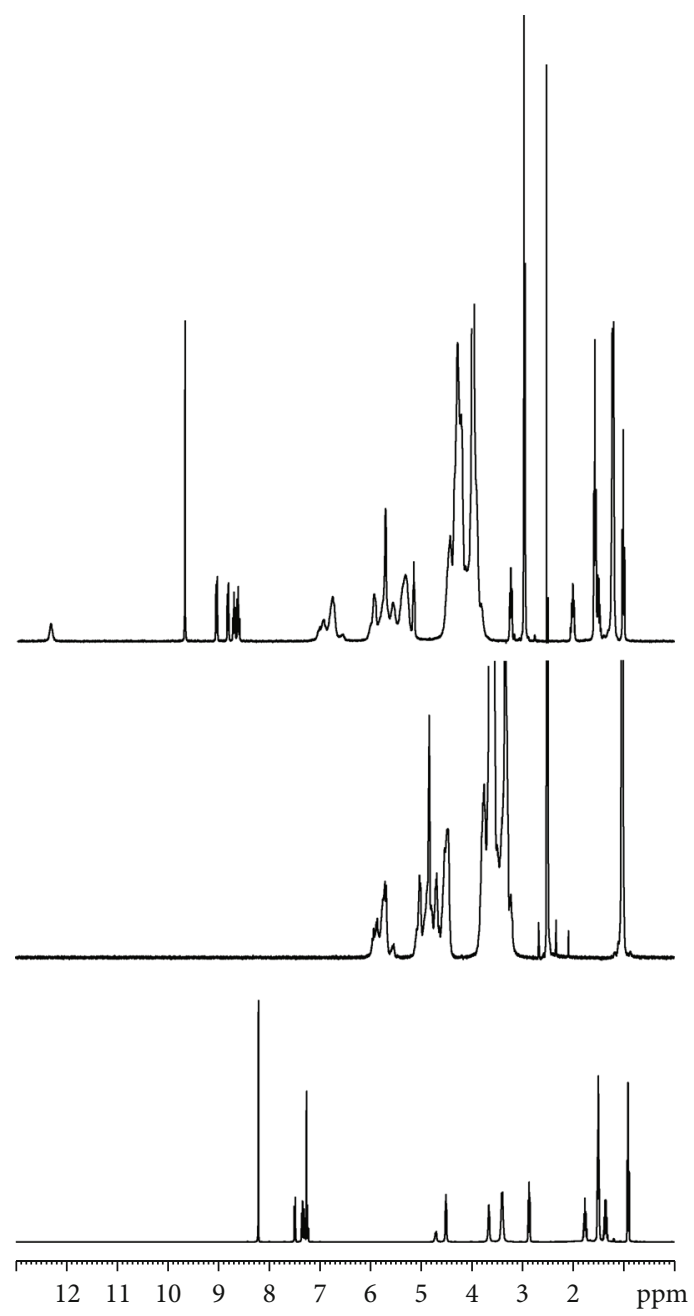

(a)

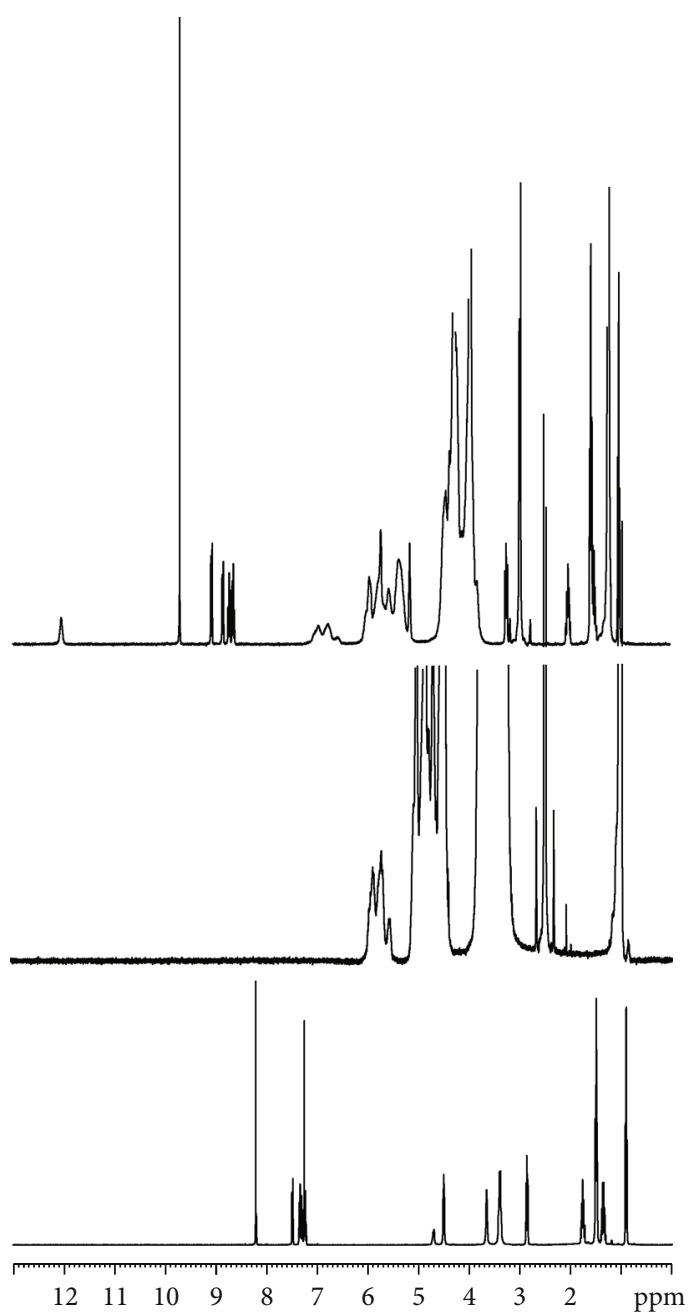

(b)

Figure 7: ${ }^{1} \mathrm{H}-\mathrm{NMR}$ spectra of the complexes AMD/HP- $\beta$-CD A and AMD/HP- $\beta$-CD B and corresponding HP- $\beta$-CDs dissolved in DMSOd6 and AMD dissolved in $\mathrm{CDCl}_{3}$-d.

AMD precipitation does not occur during dilution. The formation of the inclusion complexes was demonstrated by the increase of the solubility in water (to $0.0879 \mathrm{~g} \mathrm{~L}^{-1}$ of about 2.2 times) of AMD in the presence of $\mathrm{HP}-\beta$ $\mathrm{CD}$ in comparison with the free AMD (of $0.04 \mathrm{gL}^{-1}$ ).

The stability constants $\left(K_{\mathrm{c}}\right)$ of $\mathrm{AMD} / \mathrm{HP}-\beta-\mathrm{CD} \mathrm{A}$, $\mathrm{AMD} / \mathrm{HP}-\beta-\mathrm{CD} \mathrm{B}$, and AMD/HP- $\beta$-CD $\mathrm{C}$ complexes $(1: 1)$ were evaluated as 1218,1462 , and $1736 \mathrm{M}^{-1}$, respectively, from the linear plot of the phase solubility diagram (Figure 10). The corresponding free energy changes $(\Delta G)$ are $-17.60 \mathrm{~kJ} \mathrm{~mol}^{-1}, \quad-18.06 \mathrm{~kJ} \mathrm{~mol}^{-1}$, and $-18.48 \mathrm{~kJ} \mathrm{~mol}^{-1}$, respectively. These values are in the same limits with those found for other inclusion complexes of $\mathrm{HP}-\beta-\mathrm{CD}$ with various drugs [24].

For the three HP- $\beta$-CDs, the stability constants and negative $\Delta G$ increased with the increase in the molecular weight (degree of substitution) of the HP- $\beta$-CD and the decrease of the particle size, behavior which was also observed by Liu et al. [59]. This is probably due to the enhancement of their binding capacity with AMD as a result of the increased stability with increasing substitution by hydroxypropyl groups.
Yuan et al. [60] found that increasing substitution on the secondary hydroxyl groups of CDs could extend their cavity and thus improve the complex forming ability, which agreed with our results. The AMD/HP- $\beta$-CD B and AMD/HP- $\beta$-CD C complex seems to be more stable.

Taking into consideration the interactions between the inclusion complex partners evidenced in spectral study, the following chemical structure may be assigned to the complex (Figure 11).

Amiodarone as an iodine-rich benzofuran derivative is loaded in cavity of HP- $\beta$-CDs; its functional groups interact both with the base molecule (CD) and substituent (HP) by their $\mathrm{OH}$ groups.

3.1.6. Thermal Characterization. The DSC curves of the three complexes and their components are given in Figure 12. AMD has a particular curve, characteristic to the crystalline compounds with a sharp melting peak with a melting temperature $\left(T_{\mathrm{m}}\right)$ of $163.8^{\circ} \mathrm{C}$ and a melting heat $\left(\Delta H_{\mathrm{m}}\right)$ of $112.0 \mathrm{Jg}^{-1}$ (Table 4 ). 


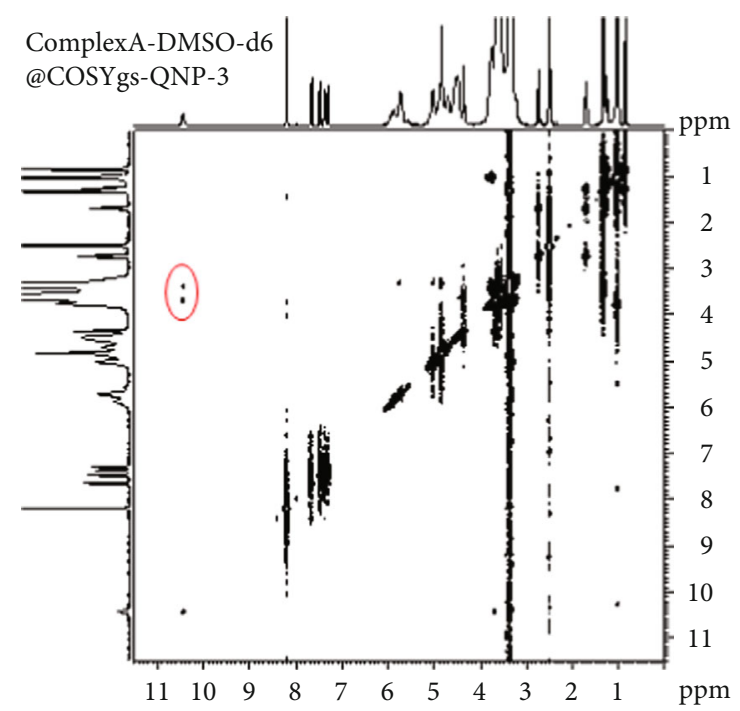

FIgURE 8: The 2D NMR spectrum of the complex A.

The thermal characteristics of the HP- $\beta$-CD A are different from those of the other two HP- $\beta$-CDs. Both $T_{\mathrm{m}}$ and $\Delta$ $H_{\mathrm{m}}$ take higher values of $89.3^{\circ} \mathrm{C}$ and $103 \mathrm{Jg}^{-1}$ while the values for HP- $\beta$-CD B and HP- $\beta$-CD C cyclodextrins are of $T_{\mathrm{m}}$ of $68^{\circ} \mathrm{C}$ and $\Delta H_{\mathrm{m}}$ of 33.8 and $40.9 \mathrm{~J} \mathrm{~g}^{-1}$, respectively (Table 4 ). The complexes have two melting peaks corresponding to both components, but they are shifted at lower temperature. The melting peak of AMD decreases from $163.8^{\circ} \mathrm{C}$ to 153 $154^{\circ} \mathrm{C}$, while those of $\mathrm{HP}-\beta$-CDs $\mathrm{A}, \mathrm{B}$, and $\mathrm{C}$ are placed at $65.5^{\circ} \mathrm{C}, 70.2^{\circ} \mathrm{C}$, and $71.4^{\circ} \mathrm{C}$, respectively. The melting heat of the first peak increases in the order complex $\mathrm{A}<$ complex $\mathrm{B}<$ complex $\mathrm{C}$ and decreases in the same order in the case of the second melting peak. This means an important decrease of the crystallinity of AMD by complexation which explains its higher solubility. This also can be explained by the particularities of each complex which determine finally their differences in solubility, loading, and release behavior of AMD.

3.1.7. Loading Degree of $A M D$ in Inclusion Complexes. In Figure 13 are presented the HPLC chromatograms of the three inclusion complexes comparatively with AMD. Comparing the peak areas, it can easily be observed that the AMD quantity included in complexes varies in the order: $\mathrm{AMD} / \mathrm{HP}-\beta$-CD $\mathrm{B}>\mathrm{AMD} / \mathrm{HP}-\beta \mathrm{A}>\mathrm{AMD} / \mathrm{HP}-\beta \mathrm{C}$. The complex $\mathrm{C}$ shows the smallest area under the curve as it includes the smallest AMD amount.

The sample concentration $\mathrm{Cp}$ in $\mathrm{mg} \mathrm{mL}^{-1}$ of $\mathrm{AMD}$ was obtained from HPLC results using the following equation:

$$
\mathrm{Cp}=\left(\text { Peak Area }+\frac{39.693}{54377}\right) \mathrm{mg} \mathrm{mL}^{-1}
$$

Complexation efficiency (CE) or loading efficacy of the complexes with AMD was evaluated as the ratio of AMD content in complexes (AMD/HP- $\beta-\mathrm{CD}$ A, AMD/HP- $\beta-\mathrm{CD}$
$\mathrm{B}$, and $\mathrm{AMD} / \mathrm{HP}-\beta-\mathrm{CD} \mathrm{C})$, determined by HPLC, to the theoretical AMD content, and was expressed in percentages by using the following relation:

$$
\% \mathrm{CE}=\left(\frac{\mathrm{AMD} \text { total }-\mathrm{AMD} \text { free }}{\mathrm{AMD} \text { total }}\right) \times 100 .
$$

The obtained results are summarized in Table 5.

The complexation efficiency is very high of 96.09-99.49\%, while the free AMD (not included in complex) is low varying in the $0.5 \%-3.91 \%$ interval. The values of the relative standard deviation (RSD) indicate that there are no significant modifications by the sample processing. Average recovery was $99.38 \%$, while recovery at the three complexes loadings is in the limits of $\pm 5 \%[39,61]$.

3.1.8. In Vitro Release Test. Release profiles of neat AMD and those incorporated into inclusion complexes at two pHs, obtained according to the above-described protocol, are given in Figure 14.

The dependence on $\mathrm{pH}$ of the dissolution/release behavior was also established in our previous paper [28] for the $\mathrm{AMD} / \mathrm{HP}-\beta-\mathrm{CD}$ system. An increase in $\mathrm{pH}$ results in the increase of the released AMD quantity from complexes especially in the first $100 \mathrm{~min}$, but not in increased solubility of pure AMD (Table 5 and Figure 14). This can be explained due to the formation of partially insoluble complex between drug and anions dissolved in buffer solution [62-64].

The release of AMD from complexes starts with a burst effect which occurs in the first 6-7 minutes, where the AMD released concentration increased very fast. The released AMD amount at the end of this stage was AMD/HP- $\beta$-CD B (7.7 $\%)>\mathrm{AMD} / \mathrm{HP}-\beta-\mathrm{CD} \mathrm{A}(5.99 \%)>\mathrm{AMD} / \mathrm{HP}-\beta-\mathrm{CD} \mathrm{C}(4.95$ $\%)>$ free $\operatorname{AMD}(3.8 \%)$ for $\mathrm{pH} 1.2$, while at $\mathrm{pH} 6.8$, the order is kept but the released amount is higher as AMD/HP- $\beta$ $\mathrm{CD} \mathrm{B}(28.2 \%)>\mathrm{AMD} / \mathrm{HP}-\beta-\mathrm{CD} \mathrm{A}(25.8 \%)>\mathrm{AMD} / \mathrm{HP}-\beta-$ CD C $(24.4 \%)>$ free AMD (4.94\%) (Table 6).

After 120 minutes, at $\mathrm{pH} 1.2$, the released AMD varies in close limits for all complexes being of AMD/HP- $\beta$-CD B ( $19.58 \%)>$ AMD $/ \mathrm{HP}-\beta$-CD A $(18.90 \%)>\mathrm{AMD} / \mathrm{HP}-\beta-\mathrm{CD} \mathrm{C}$ $(17.02 \%)$ comparative with values of only $3.8 \%$ for free AMD. This means an increase of about 5 times of drug solubility. The dissolution of free AMD of $4.25 \%$ remains almost constant for the entire studied time period while the AMD release from inclusion complexes occurred in three stages, and at the equilibrium (up to $300 \mathrm{~min}$ ), the total amount of the released AMD was AMD/HP- $\beta$-CD B $(83.7 \%)>\mathrm{AMD} /$ $\mathrm{HP}-\beta$-CD A $(78.5 \%)>\mathrm{AMD} / \mathrm{HP}-\beta$-CD C $(72.4 \%)$.

At $\mathrm{pH}$ 6.8, the release behavior is different in respect of that at $\mathrm{pH} 1.2$. The release takes place very fast in the first $2-3 \mathrm{~min}$ reaching $25-30 \%$ that means a significant burst effect, followed by a step with a very slow release rate. The three steps are also evident: the first two occurs until $120 \mathrm{~min}$ with a ADM released of about $28-35.5 \%$, and the last one took place in $120-300 \mathrm{~min}$ period, the released $A M D$ reaching about $88 \%$ a little higher than that at $\mathrm{pH}$ 1.2. The AMD released rate from complexes is higher at $\mathrm{pH} 6.8$ as a consequence of the fact that this 


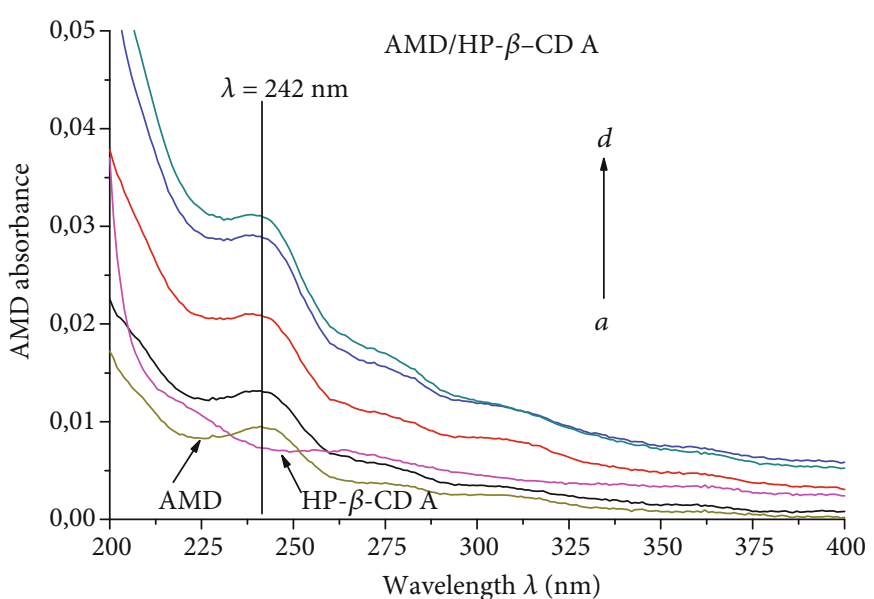

(a)

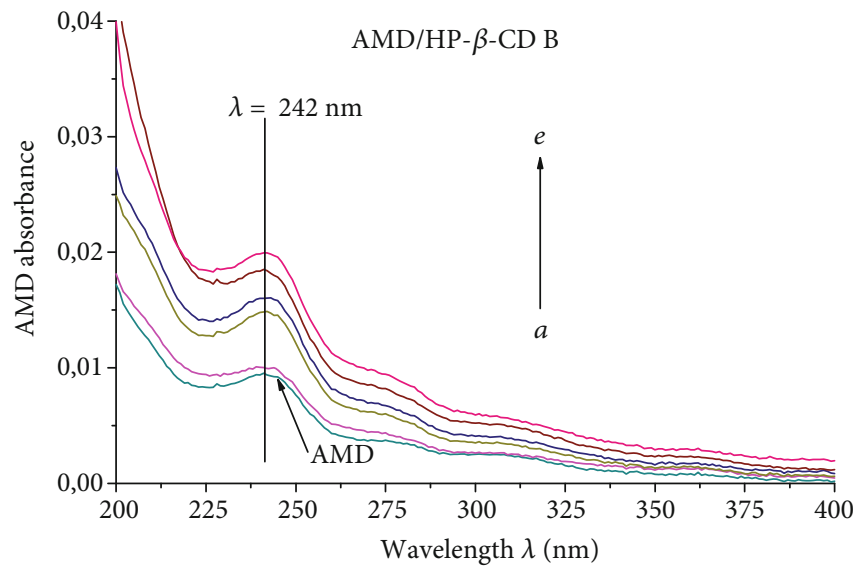

(b)

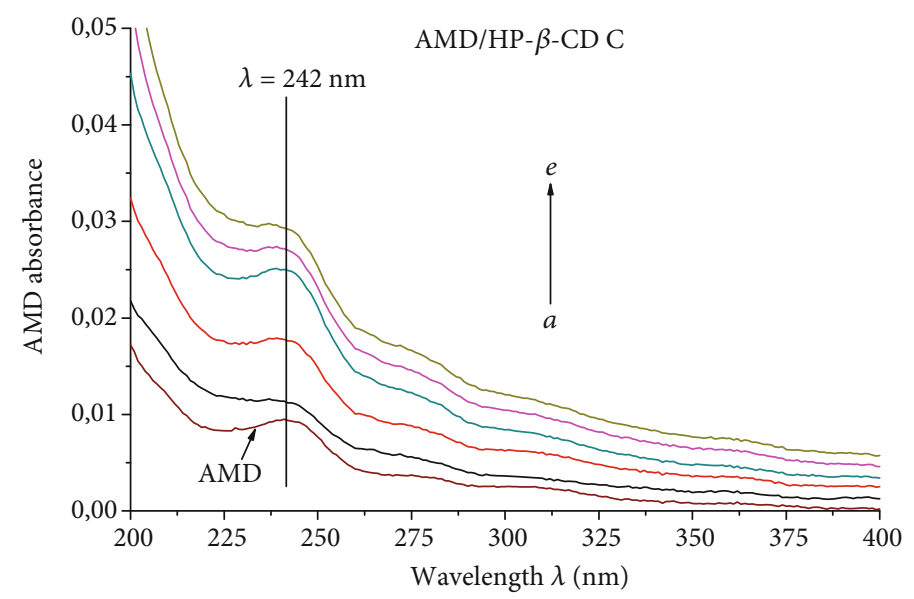

(c)

FIGURE 9: UV-VIS absorption spectra of AMD at constant concentration $\left(0.018 \times 10^{-3} \mathrm{M}\right.$ in water $)$ in the absence and presence of different concentrations of HP- $\beta$-CD. Curves $a-e$ for concentrations of $0.4,0.6,2.0,4.0$, and $6.0 \mathrm{mM}$ of HP- $\beta$-CD.

$\mathrm{pH}$ value is close of $p K_{\mathrm{a}}=6.64$ characteristic to amiodarone. The release rate from inclusion complexes increases as a consequence of the crystallinity decrease of the drug by complexation according to DSC results, which determines an increase of the solubility. At the end of the experiment, the order of the AMD released amount was AMD $/ \mathrm{HP}-\beta$-CD B $(89.2 \%)>$ AMD $/ \mathrm{HP}-\beta-\mathrm{CD} \mathrm{A}(87.5 \%)>$ $\mathrm{AMD} / \mathrm{HP}-\beta-\mathrm{CDC}(85.5 \%)$ in all cases the percentage released being higher than that at $\mathrm{pH}$ 1.2. In respect of the free AMD, an increase of $22 \%$ of AMD is released from inclusion complexes with the highest amount from the AMD/HP- $\beta$-CD B complex.

In Table 7 are reported the released kinetic parameters calculated using Equation (5) which was applied to the initial (0-50 $\mathrm{min})$, second (60-120 $\mathrm{min})$, and third (120-300 min) steps of drug release.

At $\mathrm{pH} 1.2$, a shift of the drug transport mechanism from pseudo-Fickian to non-Fickian behavior was observed between the first and the second kinetic release steps, where the values of the release exponent were under 0.5 $\left(n_{r}=0.18-0.28\right)$ in the first kinetic release step and ranged between 0.5 and $1\left(n_{r}=0.68-0.95\right)$ in the second kinetic release step (Table 7). At pH 6.8, only one type of drug transport mechanism was reported, namely, a pseudo-Fickian diffusion mechanism. A case II of the transport mechanism was observed in the third kinetic release step at $\mathrm{pH} 6.8$ $\left(n_{r}=0.93-1.00\right)$ and a special case II transport mechanism at $\mathrm{pH} 1.2\left(n_{r}=1.53-1.60\right)$. Both drug release media of $\mathrm{pH}$ 1.2 and $\mathrm{pH} 6.8$ caused the most increased values of the release exponent $\left(n_{r}\right)$ in case of the AMD/HP- $\beta$-CD B inclusion complex.

The kinetic constant $k_{r}$ values, also reported in Table 7, are higher in the case of the drug release profile of inclusion $\mathrm{AMD} / \mathrm{HP}-\beta-\mathrm{CD} \mathrm{B}$ complex, and this fact suggests that the drug diffusion degree is much increased in comparison with the inclusion AMD/HP- $\beta$-CD A complex and AMD/HP- $\beta$ CD C complex. This behavior was observed in both simulating media of $\mathrm{pH} 1.2$ and 6.8 .

The obtained values for AMD released from the three complexes after 300 minutes support the hypothesis that the AMD incorporation into inclusion complexes is a good way to realize pharmaceutical formulations with oral delivery and controlled release. If at the $\mathrm{pH} 1.2$ the solubility is still reduced, it is optimized at $\mathrm{pH} 6.8$ for such way of 


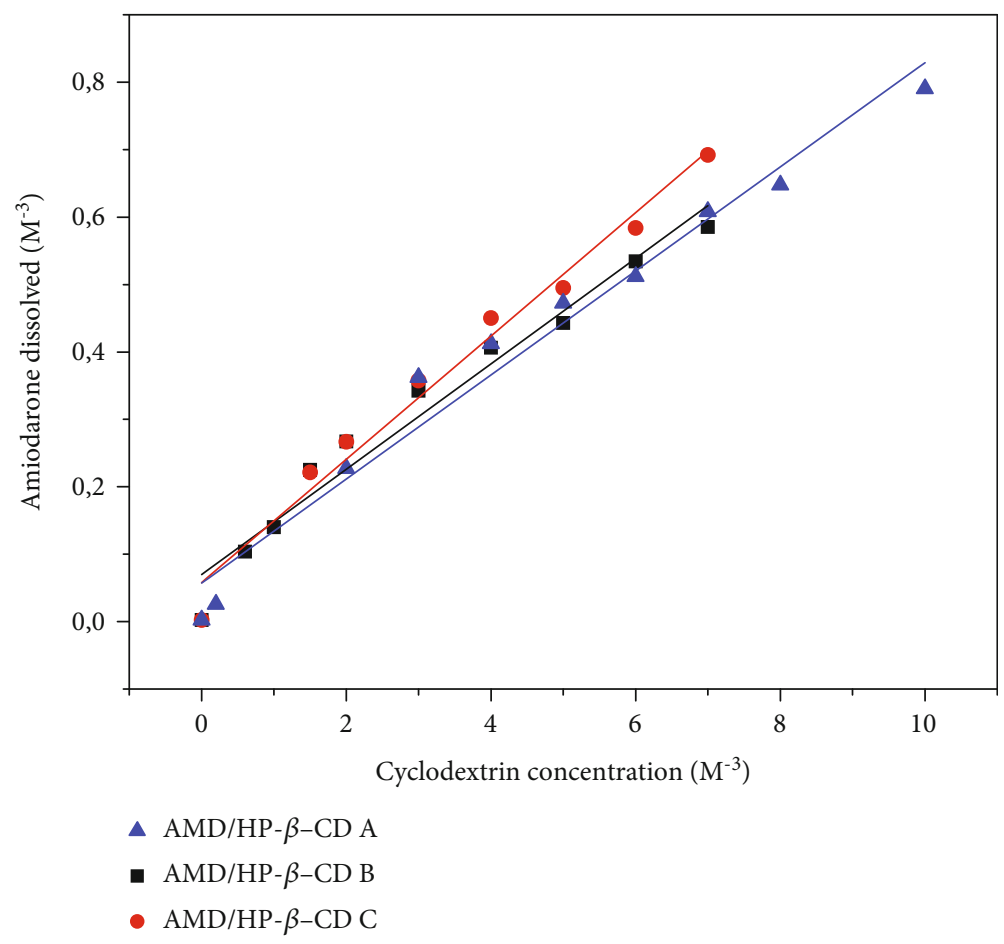

FIgURE 10: Solubility diagram of the three inclusion complexes in water: AMD/HP- $\beta$-CD A, AMD/HP- $\beta$-CD B, and AMD/HP- $\beta$-CD C.

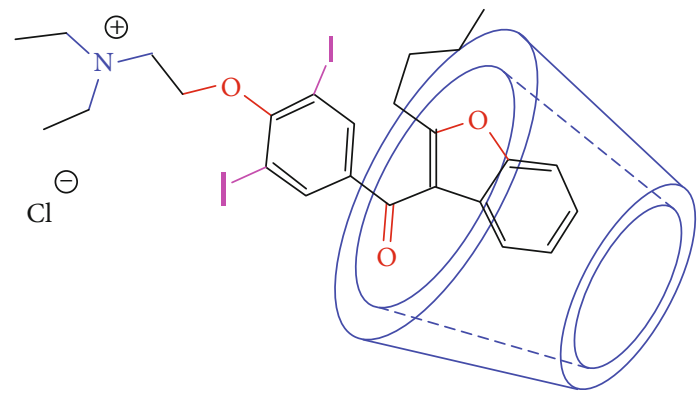

FIGURE 11: Proposed chemical structure of the inclusion complex of AMD into HP- $\beta$-CD cavity.

administration of AMD which is more convenient for patients. The prolonged release in the $6-8 \mathrm{~h}$ time interval is in accordance with optima pharmaceutical requirements for such formulations, to avoid the risk of AMD accumulation in the organism which should lead to the major toxic phenomena $[65,66]$.

\subsection{Characterization of the Matrix Tablets of AMD and Corresponding Inclusion Complex}

3.2.1. Pharmacotechnical Characterization. Pharmacotechnical characteristics given in Table 8 demonstrated that incorporation of the AMD as the inclusion complex with $\mathrm{HP}-\beta$-CD especially AMD/HP- $\beta$-CD B complex in matrix tablets based on KOL and CHT does not change the pharmacotechnical properties of the tablets. Only one relevant modification was observed in the values of the hydration degree which was found superior to Fc (124\% in respect of $115 \%$ for F). This behavior is expected to positively influence the release profile and bioavailability of the active principle.
The mass uniformity shows a deviation of $\pm 5 \%$ in respect of the mass of common tablets of $\geq 250 \mathrm{mg}$ while the dose uniformity values correspond to the individual content of active substance of each unit which should be of $85 \%$ to $115 \%$ in respect of average content. All obtained values of the pharmacotechnical characteristics are in the limits of the specifications reported in literature [40]. Friability and mechanical strength values indicate that by complexation, the AMD properties as flowability and compressibility are not changed, because between values, significant differences are not registered and these values are very close to those of the tablets with optima pharmacotechnical characteristics, namely, approximately $1 \%$ for friability and $90-100 \mathrm{~N}$ for mechanical strength. These results proved that the pharmacotechnical characteristics of the matrix tablets of $\mathrm{AMD} / \mathrm{HP}-\beta-\mathrm{CD}$ inclusion complexes are very good and the selected formulations included necessary excipients to obtain optima tablet formulations [67].

3.2.2. In Vivo Single-Dose Pharmacokinetic Study from the Matrix Tablets. Pharmacokinetic behavior as the mean plasma concentration $v s$. time curves of formulation $\mathrm{Fc}$ comparative with control samples of formulation $\mathrm{F}$ and of pure AMD suspension (corresponding to free AMD), in male Wistar rats after single oral administration of $100 \mathrm{mg} \mathrm{kg}^{-1}$, is plotted in Figure 15.

Individual values of $C_{\max }, t_{\max }$, and $\mathrm{AUC}_{0-t}$ of formulation Fc comparative with control samples of formulation $\mathrm{F}$ and of free AMD suspension from experimental data are presented in Table 9.

The areas under curves from $0 \mathrm{~h}$ to the last determined experimental data $\left(\mathrm{AUC}_{0-t}\right)$ shown in Table 10 are $3143 \pm$ $934 \mathrm{ng} \mathrm{mL}^{-1} \mathrm{~h}^{-1}$ for pure AMD suspension, $3591 \pm 1052 \mathrm{ng}$ 


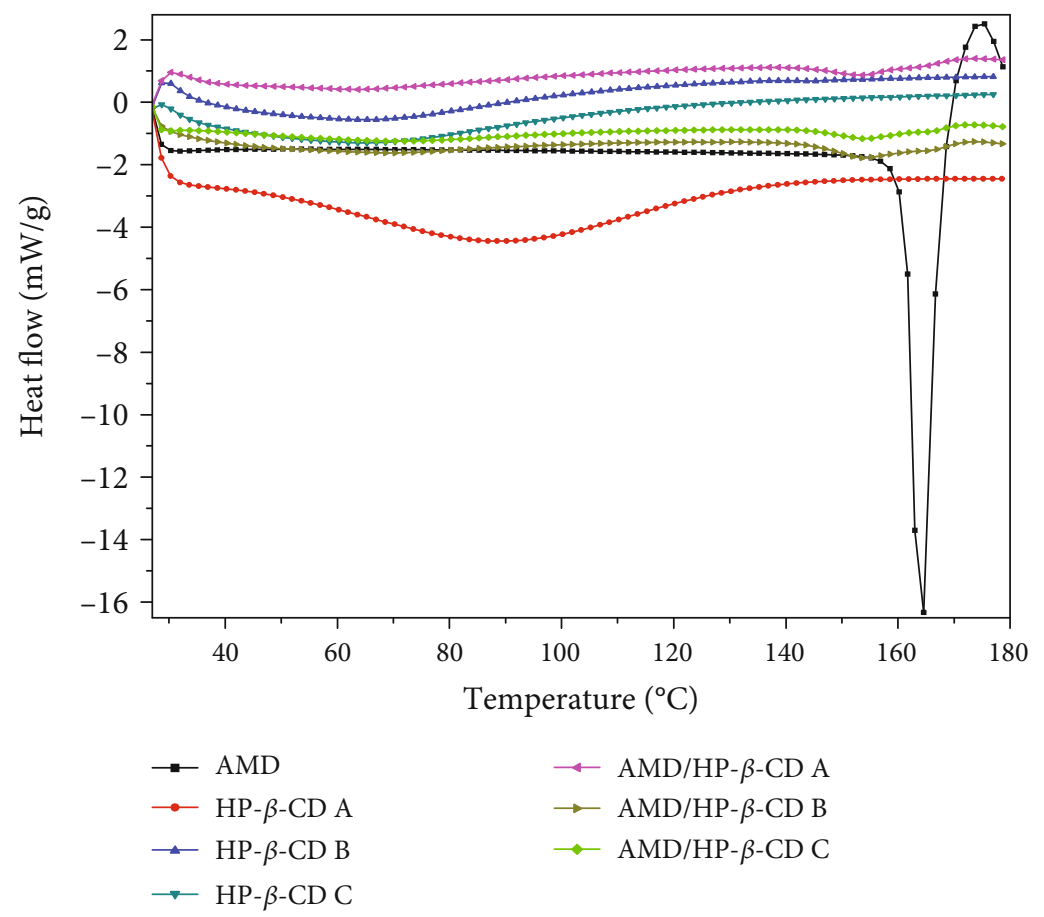

Figure 12: The DSC curves of the three AMD/HP- $\beta$-CD inclusion complexes and their components.

TABLE 4: DSC data for inclusion complexes and their components.

\begin{tabular}{lcc}
\hline Code sample & $\Delta H_{\mathrm{m}}\left(\mathrm{Jg}^{-1}\right)$ & $T_{\mathrm{m}}\left({ }^{\circ} \mathrm{C}\right)$ \\
\hline AMD & 112.0 & 163.8 \\
\hline HP- $\beta$-CD A & 103.7 & 89.3 \\
\hline HP- $\beta$-CD B & 33.8 & 68.1 \\
\hline HP- $\beta$-CD C & 40.9 & 68.1 \\
\hline \multirow{2}{*}{ AMD/HP- $\beta$-CD A } & 22.7 & 65.5 \\
& 24.2 & 153.3 \\
\hline \multirow{2}{*}{ AMD/HP- $\beta$-CD B } & 27.6 & 70.2 \\
\hline \multirow{2}{*}{ AMD/HP- $\beta$-CD C } & 19.7 & 154.0 \\
\hline
\end{tabular}

$\mathrm{mL}^{-1} \mathrm{~h}^{-1}$ for formulation $\mathrm{F}$, and $16184 \pm 2924 \mathrm{~mL}^{-1} \mathrm{~h}^{-1}$ for $\mathrm{Fc}$, a value of 5 times higher for Fc in respect of pure AMD suspension and a value of approximately 4.5 times higher for $\mathrm{Fc}$ in respect of $\mathrm{F}$.

A high value of variability ( $\mathrm{CV} \%$ ) of observed pharmacokinetic parameters is in accordance with the high interindividual variability of AMD.

Comparative pharmacokinetic parameters of formulation $\mathrm{Fc}$ in respect with control samples of formulation $\mathrm{F}$ and of free AMD are listed in Table 10.

Noncompartmental pharmacokinetic analysis was performed using TopFit 2.0 Pharmacokinetic Software (Thomae $\mathrm{GmbH}$, Germany) to determine other main pharmacokinetic parameters: area under the plasma concentration time curve from time zero to infinity $\left(\mathrm{AUC}_{0-\infty}\right)$, elimination rate constant $\left(k_{\mathrm{e}}\right)$, and half-life $\left(t_{1 / 2}\right)$. The elimi- nation rate constant $\left(k_{\mathrm{e}}\right)$ describes the rate at which a drug is removed from the body of each rat which was estimated from the slope of the regression line of plasma concentrations versus time curves by the least squares regression analysis, and the apparent elimination half-life $\left(t_{1 / 2}\right)$ was calculated from the equation:

$$
t_{1 / 2}=\frac{\ln (2)}{k_{\mathrm{e}}} .
$$

The AMD was better absorbed at the tested dose from the formulation $\mathrm{Fc}$ and poorly absorbed from the pure AMD suspension and from the $\mathrm{F} 1$ formulation. It was noted that the $C_{\max }$ obtained following the oral administration of a single dose of formulation $\mathrm{Fc}$ in rats was 1.5 and 3.8 times higher in comparison to the $C_{\max }$ obtained following formulation $\mathrm{F}$ and pure $\mathrm{AMD}$ suspension oral administration, respectively. The values of the AMD plasma concentrations released from Fc formulation independent of time belong to $<2000 \mathrm{ng} \mathrm{mL}^{-1}$, the same therapeutic interval as that found by other authors for humans $[68,69]$, and these values are similar with those reported in other studies concerning the pharmacokinetic studies using rats [70-72]. After $24 \mathrm{~h}$ from administration, the values of the plasma concentration released from Fc are also higher than those of the two control samples. This means a better absorption of AMD and a prolonged release from this new $\mathrm{Fc}$ formulation.

Further, the relative bioavailability of AMD following oral administration of formulation $\mathrm{Fc}$ was 4.5 -fold and 5.1-fold higher in Wistar rats than that of formulation $\mathrm{F}$ and pure AMD suspension, respectively. This is an 


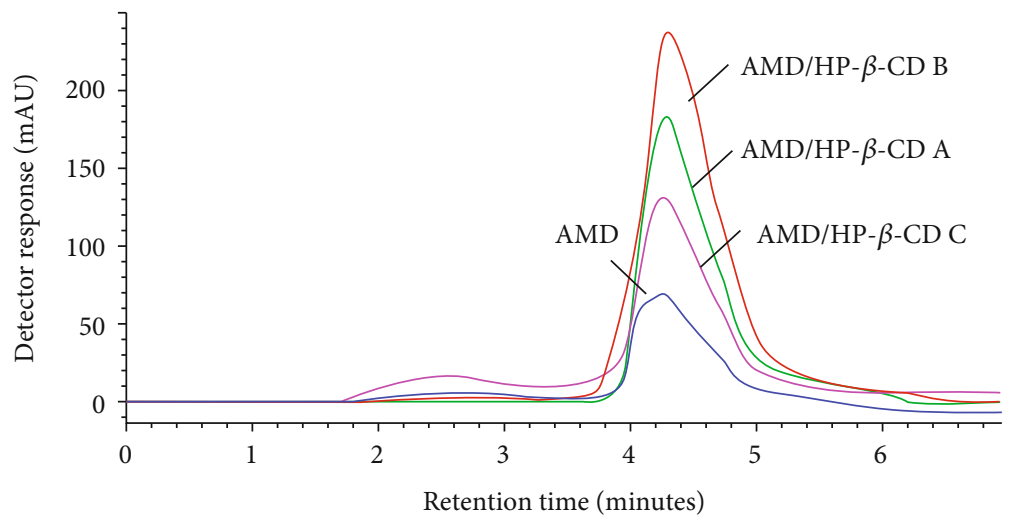

FIGURE 13: HPLC chromatograms of the three inclusion complexes: AMD/HP- $\beta$ A, AMD/HP- $\beta$ B, and AMD/HP- $\beta$ C and AMD.

TABLE 5: AMD loading efficiency in inclusion complexes.

\begin{tabular}{|c|c|c|c|c|}
\hline Inclusion complex & $\begin{array}{l}\text { Determined concentration } \\
\left(\mu \mathrm{g} \mathrm{mL}^{-1}\right)\end{array}$ & $\begin{array}{l}\text { Loading efficiency of the AMD in } \\
\text { inclusion complex CE (\%) }\end{array}$ & $\begin{array}{l}\text { Concentration of } \\
\text { the free AMD (\%) }\end{array}$ & Statistic results $n=6$ \\
\hline $\mathrm{AMD} / \mathrm{HP}-\beta-\mathrm{CD} \mathrm{A}$ & 1488 & 97.87 & 1.45 & $\begin{aligned} \mathrm{SD} & =0.373 \\
\mathrm{RSD} & =0.390 \%\end{aligned}$ \\
\hline $\mathrm{AMD} / \mathrm{HP}-\beta-\mathrm{CD} \mathrm{B}$ & 1508 & 99.49 & 0.51 & $\begin{aligned} \mathrm{SD} & =0.204 \\
\mathrm{RSD} & =0.202 \%\end{aligned}$ \\
\hline $\mathrm{AMD} / \mathrm{HP}-\beta-\mathrm{CD} \mathrm{C}$ & 1492 & 96.09 & 3.91 & $\begin{aligned} \mathrm{SD} & =0.324 \\
\mathrm{RSD} & =0.352 \%\end{aligned}$ \\
\hline Average & 1496 & Recovery $=97.96 \%$ & & \\
\hline
\end{tabular}

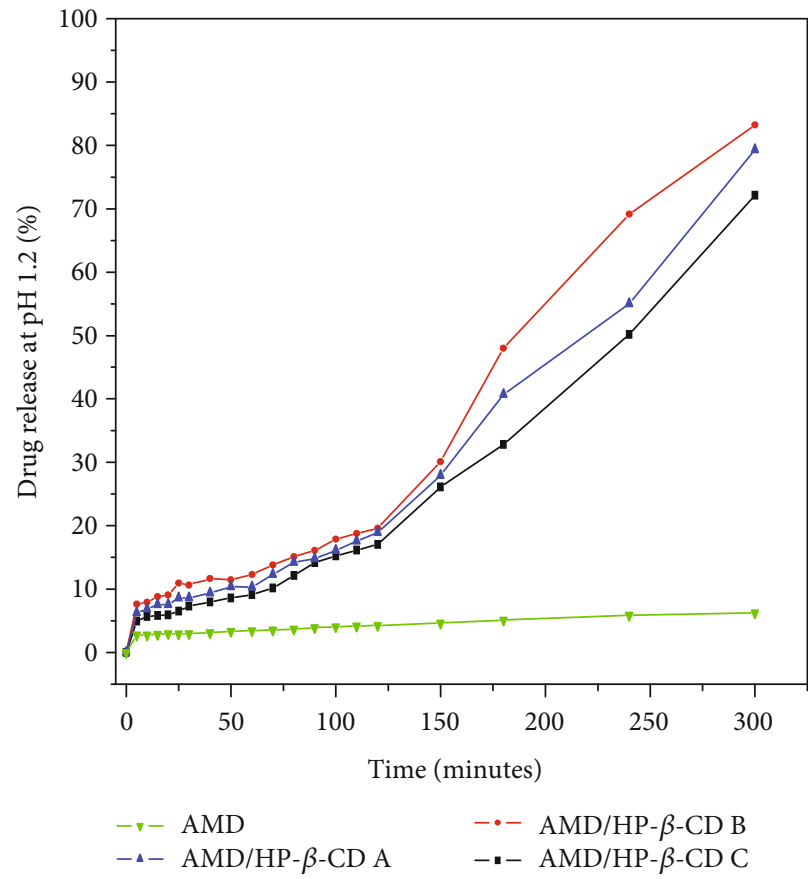

(a)

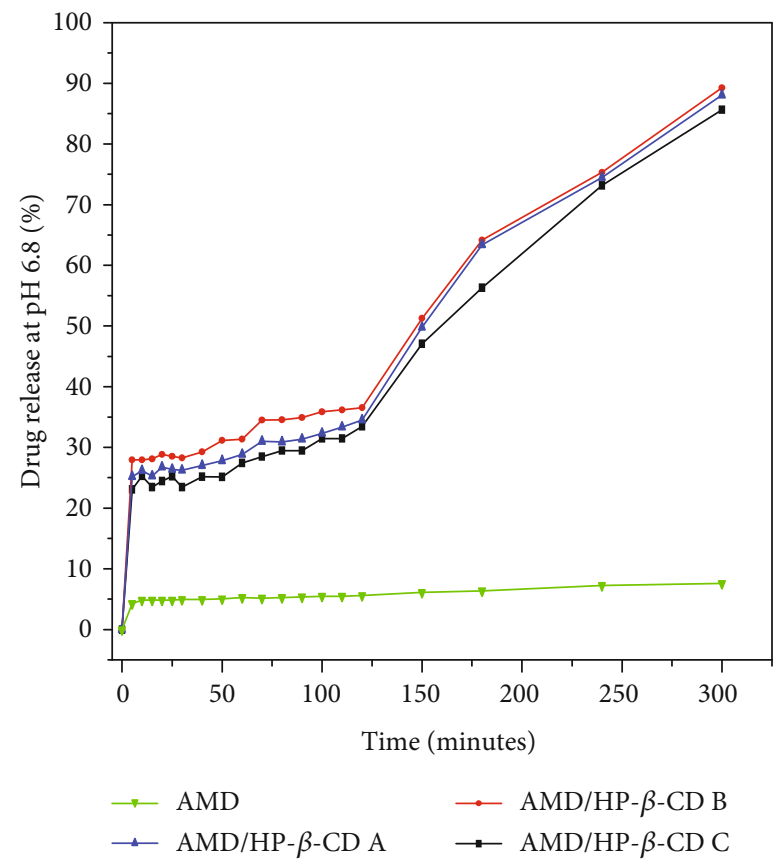

(b)

FIGURE 14: Release profiles of AMD at (a) pH 1.2 and (b) pH 6.8 from the inclusion complexes comparatively with the AMD solubility curve.

incontestable proof in favor of practical use of such matrix tablets. Relative bioavailability of $\mathrm{F}$ formulation was about 1.1 times higher compared to pure AMD suspension and also could be explained by the effect of complexation of AMD in the formulation Fc, which ultimately enhanced the digestive absorption. 
TABLE 6: Drug release parameters of the inclusion complexes (AMD/HP- $\beta$-CD A, AMD/HP- $\beta$-CD B, and AMD/HP- $\beta$-CD C) and pure AMD.

\begin{tabular}{|c|c|c|c|c|c|c|c|c|}
\hline \multirow{2}{*}{ Release characteristic } & \multicolumn{4}{|c|}{ pH 1.2} & \multicolumn{4}{|c|}{ pH 6.8} \\
\hline & $\mathrm{A}$ & B & $\mathrm{C}$ & AMD & $\mathrm{A}$ & B & $\mathrm{C}$ & AMD \\
\hline$Q_{\max }(\%)$ & 79.3 & 83.2 & 72.1 & 6.2 & 88.1 & 89.3 & 85.6 & 7.6 \\
\hline$t_{r 1 / 2}(\min )$ & 177.6 & 169.3 & 191.2 & 40.3 & 138.8 & 136.5 & 140.8 & 4.4 \\
\hline
\end{tabular}

$Q_{\max }:$ maximum release amount; $t_{\mathrm{r} 1 / 2}$ : half release time.

TABLE 7: Kinetic parameters of the AMD release from the inclusion complexes and for the AMD dissolution in the two media.

\begin{tabular}{|c|c|c|c|c|c|c|c|c|c|c|c|c|c|}
\hline \multirow[t]{2}{*}{ Samples } & \multirow[t]{2}{*}{$\mathrm{pH}$} & \multicolumn{4}{|c|}{$\begin{array}{l}\text { First step of kinetic } \\
\text { release profile }(0-50 \mathrm{~min})\end{array}$} & \multicolumn{4}{|c|}{$\begin{array}{l}\text { Second step of kinetic } \\
\text { release profile }(60-120 \mathrm{~min})\end{array}$} & \multicolumn{4}{|c|}{$\begin{array}{l}\text { Third step of kinetic release } \\
\text { profile }(120-300 \mathrm{~min})\end{array}$} \\
\hline & & $n_{r}$ & $R_{n}^{2}$ & $K(\min )^{-n} 10^{2}$ & $R_{k}^{2}$ & $n_{r}$ & $R_{n}^{2}$ & $K(\min )^{-n} 10^{3}$ & $R_{k}^{2}$ & $n_{r}$ & $R_{n}^{2}$ & $K(\min )^{-n} 10^{3}$ & $R_{k}^{2}$ \\
\hline $\mathrm{AMD} / \mathrm{HP}-\beta-\mathrm{CD} \mathrm{A}$ & 1.2 & 0.21 & 0.986 & 4.33 & 0.997 & 0.83 & 0.992 & 3.46 & 0.993 & 1.53 & 0.985 & 13.7 & 0.9885 \\
\hline $\mathrm{AMD} / \mathrm{HP}-\beta-\mathrm{CD} \mathrm{B}$ & & 0.28 & 0.986 & 4.1 & 0.998 & 0.68 & 0.997 & 7.53 & 0.997 & 1.60 & 0.953 & 51.8 & 0.939 \\
\hline $\mathrm{AMD} / \mathrm{HP}-\beta-\mathrm{CD} \mathrm{C}$ & & 0.18 & 0.934 & 3.66 & 0.993 & 0.95 & 0.990 & 1.82 & 0.988 & 1.54 & 0.995 & 2.4 & 0.998 \\
\hline AMD & & 0.05 & 0.954 & 2.55 & 0.999 & - & - & - & - & - & - & - & - \\
\hline $\mathrm{AMD} / \mathrm{HP}-\beta-\mathrm{CD} \mathrm{A}$ & 6.8 & 0.038 & 0.972 & 23.8 & 0.999 & 0.25 & 0.989 & 10.56 & 0.989 & 0.98 & 0.929 & 3.2 & 0.938 \\
\hline $\mathrm{AMD} / \mathrm{HP}-\beta-\mathrm{CD} \mathrm{B}$ & & 0.041 & 0.867 & 25.79 & 0.998 & 0.22 & 0.972 & 12.85 & 0.971 & 1.00 & 0.941 & 4.2 & 0.950 \\
\hline $\mathrm{AMD} / \mathrm{HP}-\beta-\mathrm{CD} \mathrm{C}$ & & 0.038 & 0.993 & 21.72 & 0.999 & 0.26 & 0.967 & 9.36 & 0.966 & 0.93 & 0.971 & 2.8 & 0.976 \\
\hline AMD & & 0.024 & 0.842 & 4.55 & 0.999 & - & - & - & - & - & - & - & - \\
\hline
\end{tabular}

$n_{r}$ : diffusional exponent that characterizes the drug release mechanism; $k_{r}$ : release kinetic constant; $R_{n r}{ }^{2}$ and $R_{k r}{ }^{2}:$ correlation coefficients.

TABle 8: Pharmacotechnical parameters of the matrix tablet formulations.

\begin{tabular}{lcc}
\hline $\begin{array}{l}\text { Pharmacotechnical } \\
\text { characteristics }\end{array}$ & $\begin{array}{c}\text { Formulation } \\
\mathrm{F}\end{array}$ & $\begin{array}{c}\text { Formulation } \\
\text { Fc }\end{array}$ \\
\hline Diameter (mm) & $12.076 \pm 0.010$ & $12.073 \pm 0.012$ \\
Thickness (mm) & $4.616 \pm 0.016$ & $4.625 \pm 0.030$ \\
Average mass (g) & $0.576 \pm 1.126$ & $0.595 \pm 0.530$ \\
Mass uniformity (\% minus) & -2.777 & -3.333 \\
Mass uniformity (\% plus) & +2.430 & +3.333 \\
Dose uniformity (mg/cp) & $199.42 \pm 0.9981$ & $101.71 \pm 0.596$ \\
Mechanical strength (N) & $100.10 \pm 3.334$ & $99.93 \pm 1.898$ \\
Friability (\%) & $1.036 \pm 0.019$ & $1.054 \pm 0.025$ \\
Hydration degree (\%) & 115 & 124 \\
\hline
\end{tabular}

A new modified-release formulation not only alters the release of a drug but also influence the release and dissolution rate in the different digestive sites because of different rates of absorption, leading to multiple peaking phenomena [73-75].

The double peak in the pharmacokinetic profile of the new formulation Fc (Figure 12), compared with the references pure AMD suspension and F formulation, may indicate differential absorption rates at the digestive level. These data are also in accordance with those found by in vitro study which evidences three steps of AMD release from the inclusion complexes, which could be explained both by the free AMD presence for the first stage of release as burst effect and by different kinds of bonds in complexes because of substituents and their distribution and $\beta$-CD chains. As compared to the pure AMD suspension reference, the new formulation Fc presented a 1.5-hour delay in the starting of each peak in plasma (see insert), and the amount of AMD absorbed from this new modified-release formulation is higher. The first peak of plasma concentration of AMD in the pure AMD suspension is $136.67 \pm 52.0 \mathrm{ng} \mathrm{mL}^{-1}$ and occurs at 4 hours after administration, while the first peak of plasma concentration of AMD in the formulation $\mathrm{F}$ is $186.0 \pm 56.4 \mathrm{ng} \mathrm{mL}^{-1}$ and occurs at 3 hours after administration while in the formulation $\mathrm{Fc}$, the concentration was $495.67 \pm 95.4 \mathrm{ng} \mathrm{mL}^{-1}$ and occurs at 4 hours after administration. The second peak of plasma concentration of AMD in the pure AMD suspension is $231.33 \pm 40.4 \mathrm{ng} \mathrm{mL}^{-1}$ and occurs at 4 hours after administration, while the first peak of plasma concentration of AMD in the formulation $\mathrm{F}$ is $188.0 \pm 98.0 \mathrm{ng} \mathrm{mL}^{-1}$ and occurs at 5 hours after administration and in the formulation Fc is $625.33 \pm 95.4 \mathrm{ng} \mathrm{mL}^{-1}$ and occurs at 8 hours after administration.

Following oral administration, the Fc formulation showed statistical improvement in the pharmacokinetics of AMD formulation as determined by AUC (increased by approximately 5 times, $p<0.05$ ) and $C_{\max }$ (increased by 3.6 times, $p<0.05)$. The $t_{\max }$ time needed to obtain the $C_{\max }$ of AMD was delayed for Fc formulation $\left(t_{\max }=6.3 \pm 2.0 \mathrm{~h}\right)$, a value higher than those of reference compounds pure AMD suspension $\left(t_{\max }=5.5 \pm 1.9 \mathrm{~h}\right)$ and formulation $\mathrm{F}$ $\left(t_{\max }=5.0 \pm 2.2 \mathrm{~h}\right)$, respectively. Therefore, the release of active substance from Fc formulation occurred later, but with better bioavailability than those of the reference samples.

Matrix formulation Fc containing complexed AMD enhanced the bioavailability of insoluble hydrophobic AMD by increasing the drug solubility, dissolution, and/or drug permeability, making it available at the surface of the 


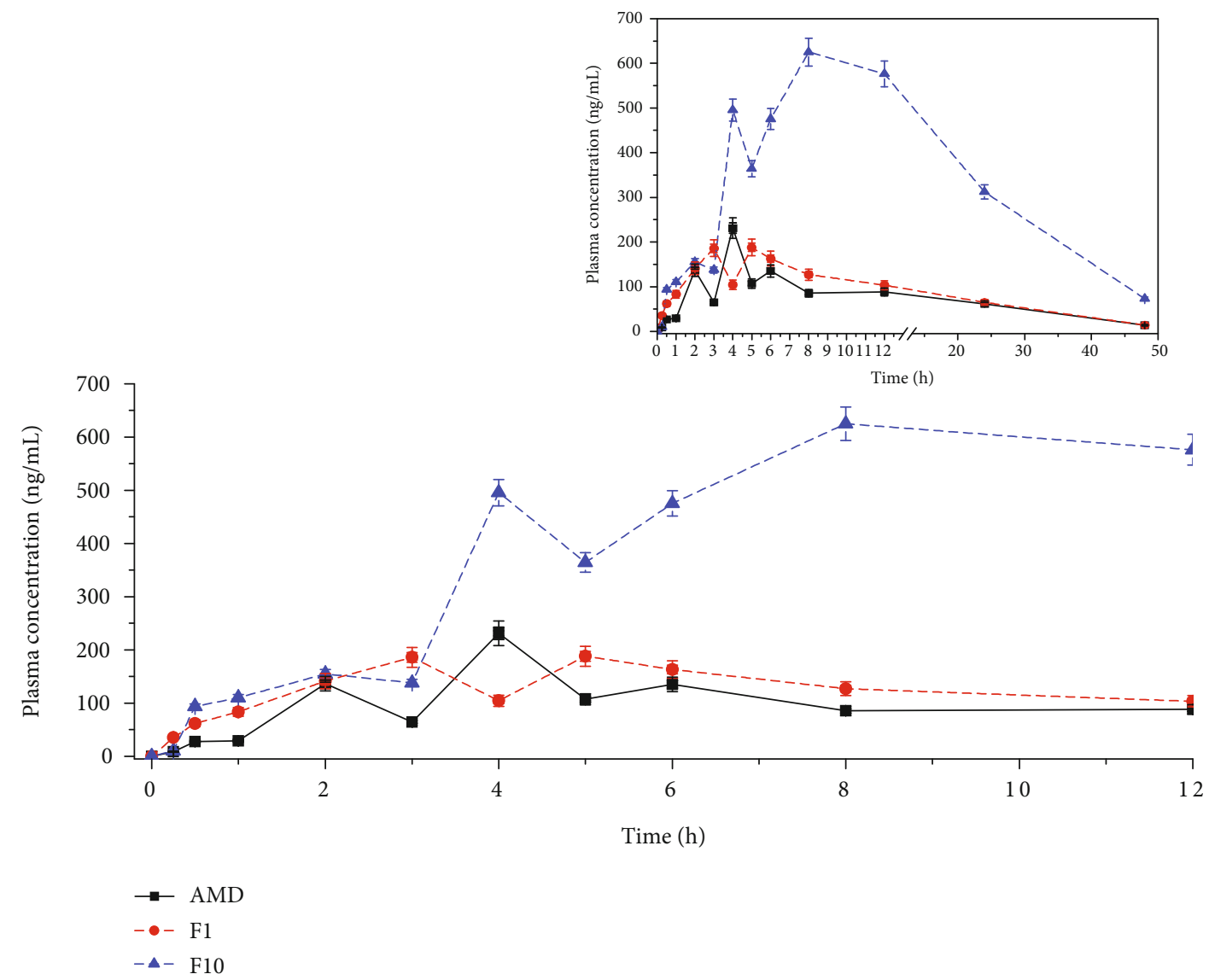

FIGURE 15: Plasma AMD concentration versus time profiles in Wistar rats following oral administration of formulation Fc, formulation F, and pure AMD suspension (mean $\pm \mathrm{SD}, n=6$ ).

TABLE 9: $C_{\max }, t_{\max }$, and $\mathrm{AUC}_{0-t}$ of pure $\mathrm{AMD}$ suspension and $\mathrm{F} 10$ and $\mathrm{F} 1$ formulations in Wistar rats following single oral administration (mean $\pm \mathrm{SD}, n=6)$.

\begin{tabular}{|c|c|c|c|c|c|c|c|c|c|}
\hline \multirow[b]{2}{*}{ Group } & \multicolumn{3}{|c|}{$C_{\max }(\mathrm{ng} / \mathrm{mL})$} & \multicolumn{3}{|c|}{$t_{\max }(\mathrm{h})$} & \multicolumn{3}{|c|}{$\operatorname{AUC}_{0-t}\left(\mathrm{mg} / \mathrm{mL}^{*} \mathrm{~h}\right)$} \\
\hline & $\begin{array}{l}\text { Pure AMD } \\
\text { suspension }\end{array}$ & $\begin{array}{c}\text { Formulation } \\
\text { F }\end{array}$ & $\begin{array}{c}\text { Formulation } \\
\text { Fc }\end{array}$ & $\begin{array}{l}\text { Pure AMD } \\
\text { suspension }\end{array}$ & $\begin{array}{c}\text { Formulation } \\
\text { F }\end{array}$ & $\begin{array}{l}\text { Formulation } \\
\text { Fc }\end{array}$ & $\begin{array}{l}\text { Pure AMD } \\
\text { suspension }\end{array}$ & $\begin{array}{c}\text { Formulation } \\
\text { F }\end{array}$ & $\begin{array}{c}\text { Formulation } \\
\text { FC }\end{array}$ \\
\hline 1 & 130 & 216 & 860 & 5 & 5 & 8 & 1373.38 & 4925.13 & 21905.88 \\
\hline 2 & 145 & 229 & 501 & 8 & 8 & 8 & 3954.50 & 3472.88 & 14752.50 \\
\hline 3 & 78 & 374 & 515 & 8 & 3 & 8 & 2072.63 & 2548.25 & 11236.25 \\
\hline 4 & 241 & 319 & 769 & 4 & 2 & 4 & 3119.00 & 2322.25 & 15540.75 \\
\hline 5 & 187 & 261 & 719 & 4 & 6 & 6 & 2829.50 & 4197.50 & 16160.00 \\
\hline 6 & 266 & 99 & 418 & 4 & 6 & 4 & 4440.00 & 2678.25 & 8889.00 \\
\hline Average & 174.50 & 249.67 & 630.33 & 5.50 & 5.00 & 6.33 & 2964.83 & 3357.38 & 14747.40 \\
\hline SD & 70.87 & 94.50 & 151.37 & 1.97 & 2.19 & 2.00 & 1142.49 & 1035.80 & 3123.50 \\
\hline CV (\%) & 40.61 & 37.85 & 24,01 & 35.91 & 43.82 & 31.58 & 38.53 & 30.85 & 21.18 \\
\hline
\end{tabular}

$C_{\max }$ : peak plasma concentration; $t_{\max }$ : time to reach peak plasma concentration; $\mathrm{AUC}_{0-t}:$ area under the curve from $0 \mathrm{~h}$ to last determined experimental data; SD: standard deviation; CV: coefficient of variation.

biological barrier, from where it partitions into the membrane without disrupting the lipid layers of the barrier. The increased drug efficacy and potency (i.e., reduction of the dose required for optimum therapeutic activity), caused by HP- $\beta$-CD presence increased drug solubility, may reduce drug toxicity by making the drug effective at lower doses. The inclusion complex of AMD with HP- $\beta$-CD might reduce the side effects by limiting the interaction and might show better tolerance with lower incidence and severity of side effects compared with the free drug. The HP- $\beta-C D$ complexation provides better absorption of drugs with poor and erratic absorption and might enhance the drug activity on oral administration. HP- $\beta$-CDs were shown a better oral safety profile. The inclusion of HP- $\beta$-CD/AMD complexes 
TABLE 10: Pharmacokinetic parameters of pure AMD suspension and $\mathrm{F}$ and Fc0 formulations in Wistar rats following single-dose oral administration (mean $\pm \mathrm{SD}, n=6)$.

\begin{tabular}{lccc}
\hline Pharmacokinetic parameter & Pure AMD suspension & Formulation F & Formulation Fc \\
\hline$C_{\max }(\mathrm{ng} / \mathrm{mL})$ & $174.50 \pm 40.61$ & $249.67 \pm 94.50^{*}$ & $630.33 \pm 151.37^{*}$ \\
$t_{\max }(\mathrm{h})$ & $5.50 \pm 1.97$ & $5.00 \pm 2.19$ & $6.33 \pm 2.00^{*}$ \\
$\mathrm{AUC}_{0-t}\left(\mathrm{ng} / \mathrm{mL}^{*} \mathrm{~h}\right)$ & $2964.83 \pm 1242.49$ & $3357 \pm 1036$ & $14747 \pm 3124^{*}$ \\
$\mathrm{AUC}_{0-\infty}\left(\mathrm{ng} / \mathrm{mL}^{*} \mathrm{~h}\right)$ & $3224.06 \pm 1202.28$ & $3591 \pm 1052$ & $16184 \pm 2924^{*}$ \\
$k_{\mathrm{e}}\left(\mathrm{h}^{-1}\right)$ & $0.053 \pm 0.01$ & $0.062 \pm 0.01$ & $0.052 \pm 0.01$ \\
$t_{1 / 2}(\mathrm{~h})$ & $12.88 \pm 0.85$ & $11.46 \pm 2.07$ & $13.71 \pm 2.47$ \\
$F_{\text {rel }}(\%)$ & & $111.4^{\mathrm{a}}$ & $501.9^{\mathrm{a}}$ \\
\hline
\end{tabular}

$C_{\max }:$ peak plasma concentration; $t_{\max }$ : time to reach peak plasma concentration; $\mathrm{AUC}_{0-t}:$ area under curve from $0 \mathrm{~h}$ to last determined experimental data; $\mathrm{AUC}_{0-\infty}$ : area under the plasma concentration time curve from time zero to infinity; $k_{\mathrm{e}}$ : elimination rate constant; $t_{1 / 2}:$ elimination half-life; $F_{\text {rel }}:$ systemic relative bioavailability. ${ }^{\mathrm{a}}$ With reference to pure AMD suspension; ${ }^{\mathrm{b}}$ with reference to formulation $\mathrm{F} 1 .{ }^{*} p<0.05$ vs. AMD.

exhibited a much higher aqueous solubility and allows also a parenteral administration of various drugs with no significant toxicity problems and hence are more often used in parenteral formulations [76-78].

$\mathrm{AMD} / \mathrm{HP}-\beta-\mathrm{CD} \mathrm{B}$ complex as such and as Fc formulation exhibited reduced drug tissue irritation and precipitation tendency because their $\mathrm{pH}$ values were significantly closer to the physiological value ( $\mathrm{pH} 7.4)$. AMD/HP- $\beta$-CD complexes are useful as slow-release carriers, in prolonged release formulations of water insoluble AMD drug.

\section{Conclusions}

In order to develop oral AMD pharmaceutical formulations with prolonged release, three inclusion complexes with different kinds of HP- $\beta$-CDs have been prepared. The HP$\beta$-CDs differ by their average molecular weight and substitution degrees. Their structural, morphological, and thermal characterization evidenced differences in particle size, types of bonding, and crystallinity on the HP- $\beta$-CD type. A substitution degree of 0.8 assured the most suitable characteristics for complex with amiodarone as stability and loading and release behavior. All studied complexes led to the increased AMD solubility and also determined different behaviors in AMD dissolution/release proved both by in vitro and by in vivo tests performed into media with two different $\mathrm{pHs}$.

It has been established that in all complexes the ratio between the two components is roughly $1: 1$. Since the loading efficacy with AMD of the three complexes varied from 96.09 to $99.49 \%$ in the order: AMD/HP- $\beta$-CD B > AMD/ $\mathrm{HP}-\beta$-CD A > AMD/HP- $\beta$-CD C; the highest efficacy was found for the AMD/HP- $\beta$-CD B complex containing 2-HP$\beta$-CD with the degree of substitution 0.8 and molecular weight $1460 \mathrm{Da}$. The AMD release from inclusion complexes occurred at the higher amount up to $22 \%$ times more than that from free AMD.

Matrix tablets containing Kollidon ${ }^{\circledR}$ and chitosan as available and cheap excipients have been obtained by direct compression using high amounts of complex $(200 \mathrm{mg} / \mathrm{matrix}$ tablet). Such matrix tablets showed a prolonged and sustain modified release and a bioavailability of 5 times higher than those of conventional tablets. This offers a prolonged oral delivery at the plasmatic concentrations belonging to the efficient therapeutic concentrations.

The formulations of AMD/HP- $\beta$-CD inclusion complexes both from powdered form and from matrix tablets showed superior pharmacokinetic performance by improving loading and release properties of the insoluble AMD drug. In vitro kinetic study revealed a complex mechanism of release occurring in three steps: the first one being attributed to a burst effect and the other two to different bonding in inclusion complexes. The in vivo test on matrix tablets containing Kollidon ${ }^{\circledR}$ and chitosan, similarly, shows significant difference between the plasma drug levels over time between the free drugs as pure AMD suspension and the new formulation, also being characterized by multiple peak plasma concentrations because of both structures of the inclusion complexes which determined prolonged and increased releases of AMD and also of different sites of absorption in biological media (digestive tract).

\section{Data Availability}

Data available on request

\section{Conflicts of Interest}

The authors declare that there is no conflict of interest regarding the publication of this paper.

\section{Authors' Contributions}

All authors contributed equally to this work.

\section{Acknowledgments}

The authors thank Dr. Oana-Maria Mocanu for valuable support on helping us to conduct some experiments. The research leading to these results has received funding from 
Universitatea de Medicina și Farmacie Grigore T. Popa - Iasi through the project 29025/2016.

\section{References}

[1] A. Laina, G. Karlis, A. Liakos et al., "Amiodarone and cardiac arrest: systematic review and meta-analysis," International Journal of Cardiology, vol. 221, pp. 780-788, 2016.

[2] H. Van Herendael and P. Dorian, "Amiodarone for the treatment and prevention of ventricular fibrillation and ventricular tachycardia," Vascular Health and Risk Management, vol. 6, pp. 465-472, 2010.

[3] J. Cohen-Lehman, P. Dahl, S. Danzi, and I. Klein, "Effects of amiodarone therapy on thyroid function," Nature Reviews Endocrinology, vol. 6, no. 1, pp. 34-41, 2010.

[4] J. T. Lu, Y. Cai, F. Chen, W. W. Jia, Z. Y. Hu, and Y. S. Zhao, “A physiologically based pharmacokinetic model of amiodarone and its metabolite desethylamiodarone in rats: pooled analysis of published data," European Journal of Drug Metabolism and Pharmacokinetics, vol. 41, no. 6, pp. 689-703, 2016.

[5] R. Latini, G. Tognoni, and R. E. Kates, "Clinical pharmacokinetics of amiodarone," Clinical Pharmacokinetics, vol. 9, no. 2, pp. 136-156, 1984.

[6] I. Atanasova and D. Terziivanov, "Evaluation of average bioequivalence of two oral formulations of amiodarone hydrochloride after single administration to healthy volunteers," Clinical Drug Investigation, vol. 21, no. 6, pp. 423-428, 2001.

[7] E. Hrudikova Vyskocilova, M. Grundmann, J. Duricova, and I. Kacirova, "Therapeutic monitoring of amiodarone: pharmacokinetics and evaluation of the relationship between effect and dose/concentration," Biomedical Papers, vol. 161, no. 2, pp. 134-143, 2017.

[8] A. Elgart, I. Cherniakov, Y. Aldouby, A. J. Domb, and A. Hoffman, "Improved oral bioavailability of BCS class 2 compounds by self nano-emulsifying drug delivery systems (SNEDDS): the underlying mechanisms for amiodarone and talinolol," Pharmaceutical Research, vol. 30, no. 12, pp. 30293044, 2013.

[9] J. Emami, "Comparative in vitro and in vivo evaluation of three tablet formulations of amiodarone in healthy subjects," Daru, vol. 18, no. 3, pp. 193-199, 2010.

[10] W. Dai, C. Pollock-Dove, L. C. Dong, and S. Li, “Advanced screening assays to rapidly identify solubility-enhancing formulations: high-throughput, miniaturization and automation," Advanced Drug Delivery Reviews, vol. 60, no. 6, pp. 657-672, 2008.

[11] M. N. Reddy, T. Rehana, S. Ramakrishna, K. P. R. Chowdary, and P. V. Diwan, " $\beta$-Cyclodextrin complexes of celecoxib: molecular-modeling, characterization, and dissolution studies," AAPS PharmSci, vol. 6, no. 1, pp. 68-76, 2004.

[12] G. M. Khan, F. Wazir, and J.-b. Zhu, "Ibuprofen-cyclodextrin inclusion complexes: evaluation of different complexation methods," Journal of Medical Sciences(Faisalabad), vol. 1, no. 4, pp. 193-199, 2001.

[13] L. P. Ruan, B. Y. Yu, G. M. Fu, and D. N. Zhu, "Improving the solubility of ampelopsin by solid dispersions and inclusion complexes," Journal of Pharmaceutical and Biomedical Analysis, vol. 38, no. 3, pp. 457-464, 2005.

[14] M. K. Riekes, M. P. Tagliari, A. Granada, G. Kuminek, M. A. S. Silva, and H. K. Stulzer, "Enhanced solubility and dissolution rate of amiodarone by complexation with $\beta$-cyclodextrin through different methods," Materials Science and Engineering: $C$, vol. 30, no. 7, pp. 1008-1013, 2010.

[15] F. Cramer, W. Saenger, and H. C. Satz, "Inclusion compounds. XIX.1aThe formation of inclusion compounds of $\alpha$-Cyclodextrin in aqueous Solutions. thermodynamics and kinetics," Journal of the American Chemical Society, vol. 89, no. 1, pp. 14-20, 1967.

[16] R. Challa, A. Ahuja, J. Ali, and R. K. Khar, "Cyclodextrins in drug delivery: an updated review," AAPS PharmSciTech, vol. 6, no. 2, pp. E329-E357, 2005.

[17] L. Szente and J. Szejtli, "Highly soluble cyclodextrin derivatives: chemistry, properties, and trends in development," Advanced Drug Delivery Reviews, vol. 36, no. 1, pp. 17-28, 1999.

[18] H. Matsuda and H. Arima, "Cyclodextrins in transdermal and rectal delivery," Advanced Drug Delivery Reviews, vol. 36, no. 1, pp. 81-99, 1999.

[19] S. Gould and R. Scott, "2-Hydroxypropyl- $\beta$-cyclodextrin (HP$\beta$-CD): a toxicology review," Food and Chemical Toxicology, vol. 43, no. 10, pp. 1451-1459, 2005.

[20] S. Jambhekar, R. Casella, and T. Maher, "The physicochemical characteristics and bioavailability of indomethacin from $\beta$ cyclodextrin, hydroxyethyl- $\beta$-cyclodextrin, and hydroxypropyl- $\beta$-cyclodextrin complexes," International Journal of Pharmaceutics, vol. 270, no. 1-2, pp. 149-166, 2004.

[21] A. Sherje and V. Londhe, "Inclusion complexes of hydroxy propyl- $\beta$-cyclodextrin and paliperidone: preparation and characterization," Current Drug Discovery Technologies, vol. 11, no. 4, pp. 271-278, 2014.

[22] S. Wang, Y. Ding, and Y. Yao, "Inclusion complexes of fluorofenidone with $\beta$-cyclodextrin and hydroxypropyl- $\beta$-cyclodextrin," Drug Development and Industrial Pharmacy, vol. 35, no. 7, pp. 808-813, 2009.

[23] H.-G. Choi, D.-D. Kim, H. Won Jun, B.-K. Yoo, and C.S. Yong, "Improvement of dissolution and bioavailability of nitrendipine by inclusion in hydroxypropyl- $\beta$-cyclodextrin," Drug Development and Industrial Pharmacy, vol. 29, no. 10, pp. 1085-1094, 2003.

[24] U. Domańska, A. Pelczarska, and A. Pobudkowska, "Effect of 2-hydroxypropyl- $\beta$-cyclodextrin on solubility of sparingly soluble drug derivatives of anthranilic acid," International Journal of Molecular Sciences, vol. 12, no. 4, pp. 23832394, 2011.

[25] C. Jantarat, P. Sirathanarun, S. Ratanapongsai, P. Watcharakan, S. Sunyapong, and A. Wadu, "CurcuminHydroxypropyl- $\beta$-Cyclodextrin Inclusion Complex Preparation Methods: Effect of Common Solvent Evaporation, Freeze Drying, and pH Shift on Solubility and Stability of Curcumin," Tropical Journal of Pharmaceutical Research, vol. 13, no. 8, pp. 1215-1223, 2014.

[26] J. Pitha, J. Milecki, H. Fales, L. Pannell, and K. Uekama, "Hydroxypropyl- $\beta$-cyclodextrin: preparation and characterization; effects on solubility of drugs," International Journal of Pharmaceutics, vol. 29, no. 1, pp. 73-82, 1986.

[27] A. M. Rubim, J. B. Rubenick, M. Maurer, L. V. Laporta, and C. M. B. Rolim, "Inclusion complex of amiodarone hydrochloride with cyclodextrins: preparation, characterization and dissolution rate evaluation," Brazilian Journal of Pharmaceutical Sciences, vol. 53, no. 2, 2017.

[28] O. M. Păduraru, A. Bosînceanu, G. Țântaru, and C. Vasile, "Effect of Hydroxypropyl- $\beta$-Cyclodextrin on the solubility of 
an Antiarrhythmic agent," Industrial and Engineering Chemistry Research, vol. 52, no. 5, pp. 2174-2181, 2013.

[29] A. Creteanu, L. Ochiuz, C. Vasile et al., "Thermal stability assesment of amiodarone hydrochloride in polymeric matrix tablets," Farmacia, vol. 64, no. 6, 2016.

[30] A. Creteanu, L. Ochiuz, C. Vasile, M. Vieriu, and G. Tantaru, "Studies on the influence of amiodarone complexation with cyclodextrin derivatives on the in vitro release from matrix tablets," Farmacia, vol. 65, no. 4, pp. 545-549, 2017.

[31] A. Crețeanu, L. Ochiuz, M. Vieriu, A. D. Panainte, and G. Tantaru, "In vitro dissolution studies of amiodarone hydrochloride From hydroxy-propyl- $\beta$-cyclodextrin/amiodarone inclusion complex formulated into modified-release tablets," Revista medico-chirurgicala a Societatii de Medici si Naturalisti din Iasi, vol. 120, no. 3, pp. 715-719, 2016.

[32] J. A. Castillo, J. Palomo-Canales, J. J. Garcia, J. L. Lastres, F. Bolas, and J. J. Torrado, "Preparation and characterization of albendazole beta-cyclodextrin complexes," Drug Development and Industrial Pharmacy, vol. 25, no. 12, pp. 12411248, 1999.

[33] D. Díaz, C. M. E. Llanos, and M. J. B. Bernad, "Study of the binding in an aqueous medium of inclusion complexes of several cyclodextrins involving fenoprofen calcium," Drug Development and Industrial Pharmacy, vol. 25, no. 1, pp. 107-110, 1999.

[34] V. Bühler, "Polyvinylpyrrolidone excipients for the pharmaceutical industry, 9th revised edition," in Ludwigshafen: BASF SE Pharma Ingredients \& Services, pp. 255-270, Springer Berlin, Heidelberg, Germany, 2008.

[35] M. M. Thanou, A. F. Kotzé, T. Scharringhausen et al., "Effect of degree of quaternization of_N_-trimethyl chitosan chloride for enhanced transport of hydrophilic compounds across intestinal Caco-2 cell monolayers," Journal of Controlled Release, vol. 64, no. 1-3, pp. 15-25, 2000.

[36] R. A. A. Muzzarelli and C. Muzzarelli, "Chitosan chemistry: relevance to the biomedical sciences," in Polysaccharides I, pp. 151-209, Springer, Berlin, Heidelberg.

[37] S. Niazi, Handbook of Pharmaceutical Manufacturing Formulations: Compressed Solid Products, vol. 1, Informa Health Care, CRC Press, New York, USA, 2nd edition, 2009.

[38] T. Higuchi and K. A. Connors, "Phase-solubility techniques," in Advances in Analytical Chemistry and Instrumentation, C. N. Reilly, Ed., vol. 4, pp. 117-212, Wiley-Interscience, New York, USA, 1965.

[39] A. Bosînceanu, O. M. Păduraru, C. Vasile, I. Popovici, G. Țântaru, and L. Ochiuz, "Validation of a new HPLC method used for determination of amiodarone from the complex with hydroxypropyl-beta-cyclodextrin and from comercial tablets," Farmácia, vol. 61, no. 5, pp. 856-864, 2013.

[40] "Farmacopeea Română," in Ed. Medicală, a X a Edition, pp. 59-74, Bucharest, 2004.

[41] European Pharmacopoeia, Directorate for the Quality of Medicines and Healthcare, Council of Europe, Strasbourg, 8th ed edition, 2014.

[42] "The official compendia of standards," in United States Pharmacopoeia and the National Formulary, US Pharmacopoeia Convention, Inc, 26th edition, 2003.

[43] R. W. Korsmeyer, S. R. Lustig, and N. A. Peppas, "Solute and penetrant diffusion in swellable polymers. I. Mathematical modeling," Journal of Polymer Science Part B: Polymer Physics, vol. 24, no. 2, pp. 395-408, 1986.
[44] E. B. Denkbas and R. M. Ottenbrite, "Perspectives on: chitosan drug delivery systems based on their geometries," Journal of Bioactive and Compatible Polymers, vol. 21, no. 4, pp. 351368, 2006.

[45] P. Thapa, M. Ghimire, A. B. Mullen, and H. Stevens, "Controlled release oral drug delivery systems containing water insoluble drugs," Journal of Science, Engineering and Technology, vol. 1, pp. 28-35, 2005.

[46] M. C. Gohel, K. G. Sarvaiya, A. R. Shah, and B. K. Brahmbhatt, "Mathematical approach for the assessment of similarity factor using a new scheme for calculating weight," Indian Journal of Pharmaceutical Sciences, vol. 71, no. 2, pp. 142-144, 2009.

[47] R. Herbois, S. Noël, B. Léger et al., "Cyclodextrins as growth controlling agents for enhancing the catalytic activity of PVP-stabilized $\mathrm{Ru}(0)$ nanoparticles," Chemical Communications, vol. 48, no. 28, p. 3451, 2012.

[48] M. Messner, S. V. Kurkov, M. M. Palazón, B. Á. Fernández, M. E. Brewster, and T. Loftsson, "Self-assembly of cyclodextrin complexes: effect of temperature, agitation and media composition on aggregation," International Journal of Pharmaceutics, vol. 419, no. 1-2, pp. 322-328, 2011.

[49] G. Gonzalez-Gaitano, P. Rodriguez, J. R. Isasi, M. Fuentes, G. Tardajos, and M. Sanchez, "The aggregation of cyclodextrins as studied by photon correlation spectroscopy," Journal of Inclusion Phenomena and Macrocyclic Chemistry, vol. 44, no. 1/4, pp. 101-105, 2002.

[50] C. Yuan, B. Liu, and H. Liu, "Characterization of hydroxypropyl- $\beta$-cyclodextrins with different substitution patterns via FTIR, GC-MS, and TG-DTA," Carbohydrate Polymers, vol. 118, pp. 36-40, 2014.

[51] D. Wang, G. Chen, and L. Ren, "Preparation and characterization of the sulfobutylether- $\beta$-cyclodextrin inclusion complex of amiodarone hydrochloride with enhanced oral bioavailability in fasted state," AAPS PharmSciTech, vol. 18, no. 5, pp. 1526-1535, 2017.

[52] K. P. Sambasevam, S. Mohamad, N. M. Sarih, and N. A. Ismail, "Synthesis and characterization of the inclusion complex of $\beta$ cyclodextrin and azomethine," International Journal of Molecular Sciences, vol. 14, no. 2, pp. 3671-3682, 2013.

[53] B. Tang, Z.-Z. Chen, N. Zhang, J. Zhang, and Y. Wang, "Synthesis and characterization of a novel cross-linking complex of $\beta$-cyclodextrin-_o_-vanillin furfuralhydrazone and highly selective spectrofluorimetric determination of trace gallium," Talanta, vol. 68, no. 3, pp. 575-580, 2006.

[54] H. Hamdi, R. Abderrahim, and F. Meganem, "Spectroscopic studies of inclusion complex of $\beta$-cyclodextrin and benzidine diammonium dipicrate," Spectrochimica Acta Part A: Molecular and Biomolecular Spectroscopy, vol. 75, no. 1, pp. 32-36, 2010.

[55] C. Nicolescu, C. Arama, and C. M. Monciu, "Preparation and characterization of inclusion complexes between repaglinide and $\beta$-cyclodextrin, 2 -hydroxypropyl- $\beta$-cyclodextrin and randomly methylated $\beta$-cyclodextrin," Farmacia, vol. 58 , no. 1 , pp. 78-88, 2010.

[56] C. M. Fernandes, R. A. Carvalho, S. P. da Costa, and F. J. Veiga, "Multimodal molecular encapsulation of nicardipine hydrochloride by $\beta$-cyclodextrin, hydroxypropyl- $\beta$-cyclodextrin and triacetyl- $\beta$-cyclodextrin in solution. Structural studies by ${ }^{1} \mathrm{H}$ NMR and ROESY experiments," European Journal of Pharmaceutical Sciences, vol. 18, no. 5, pp. 285-296, 2003.

[57] S. Barman, B. K. Barman, and M. N. Roy, "Preparation, characterization and binding behaviors of host-guest inclusion 
complexes of metoclopramide hydrochloride with $\alpha \backslash$ - and $\beta$ cyclodextrin molecules," Journal of Molecular Structure, vol. 1155, pp. 503-512, 2018.

[58] B. W. Muller and U. Brauns, "Hydroxypropyl- _ $\beta$ _ cyclodextrin derivatives: influence of average degree of substitution on complexing ability and surface activity," Journal of Pharmaceutical Sciences, vol. 75, no. 6, pp. 571-572, 1986.

[59] B. Liu, W. Li, T. A. Nguyen, and J. Zhao, "Empirical, thermodynamic and quantum-chemical investigations of inclusion complexation between flavanones and (2-hydroxypropyl)cyclodextrins," Food Chemistry, vol. 134, no. 2, pp. 926-932, 2012.

[60] C. Yuan, Z. Jin, and X. Li, "Evaluation of complex forming ability of hydroxypropyl- $\beta$-cyclodextrins," Food Chemistry, vol. 106, no. 1, pp. 50-55, 2008.

[61] M. Yowono and G. Indryanoto, "Validation of chromatographic methods of analysis," in Profiles of Drug Substances, Excipients and Related Methology, H. Brittain, Ed., vol. 32pp. 243-262, Academic Press, Waltham, 1 edition, 2005.

[62] A. Avdeef, "Solubility of sparingly-soluble ionizable drugs," Advanced Drug Delivery Reviews, vol. 59, no. 7, pp. 568-590, 2007.

[63] F. Boury, J.-C. Gautier, Y. Bouligand, and J.-E. Proust, "Interfacial properties of amiodarone: the stabilizing effect of phosphate anions," Colloids and Surfaces B: Biointerfaces, vol. 20, no. 3, pp. 219-227, 2001.

[64] L. J. Ravin, E. G. Shami, and E. S. Rattie, "Micelle formation and its relationship to solubility behavior of 2-butyl-3-benzofuranyl-4-[2-(diethylamino)ethoxy]-3,5-diiodophenyl ketone hydrochloride," Journal of Pharmaceutical Sciences, vol. 64, no. 11, pp. 1830-1833, 1975.

[65] G. Vasvári, J. Kalmár, P. Veres et al., "Matrix systems for oral drug delivery: formulations and drug release," Drug Discovery Today: Technologies, vol. 27, pp. 71-80, 2018.

[66] S. Maqdasy, T. Benichou, S. Dallel et al., "Issues in amiodarone-induced thyrotoxicosis: Update and review of the literature," in Annales d'endocrinologie, vol. 80, no. 1pp. 5460, Elsevier Masson, 2019.

[67] I. Popovici and D. Lupuleasa, Tehnologie Farmaceutică, vol. 3, Polirom Iași, 2017.

[68] A. Shayeganpour, D. A. Hamdy, and D. R. Brocks, "Pharmacokinetics of desethylamiodarone in the rat after its administration as the preformed metabolite, and after administration of amiodarone," Biopharmaceutics \& Drug Disposition, vol. 29, no. 3, pp. 159-166, 2008.

[69] I. Fabiani, D. Tacconi, S. Grotti et al., "Amiodarone-induced pulmonary toxicity mimicking acute pulmonary edema," Journal of Cardiovascular Medicine, vol. 12, no. 5, pp. 361-365, 2011.

[70] M. Rodrigues, G. Alves, J. Abrantes, and A. Falcão, "Herb-drug interaction of_Fucus vesiculosus_extract and amiodarone in rats: a potential risk for reduced bioavailability of amiodarone in clinical practice," Food and Chemical Toxicology, vol. 52, pp. 121-128, 2013.

[71] M. Rodrigues, G. Alves, J. Francisco, A. Fortuna, and A. Falcão, "Herb-drug pharmacokinetic interaction between Carica papaya extract and amiodarone in rats," Journal of Pharmacy \& Pharmaceutical Sciences, vol. 17, no. 3, pp. 302-315, 2014.

[72] C. Thapa, A. Ahad, M. Aqil, S. S. Imam, and Y. Sultana, "Formulation and optimization of nanostructured lipid carriers to enhance oral bioavailability of telmisartan using Box-Behnken design," Journal of Drug Delivery Science and Technology, vol. 44, pp. 431-439, 2018.

[73] N. M. Davies, J. K. Takemoto, D. R. Brocks, and J. A. Yáñez, "Multiple peaking phenomena in pharmacokinetic disposition," Clinical Pharmacokinetics, vol. 49, no. 6, pp. 351-377, 2010.

[74] A. Mittur, S. Gupta, and N. B. Modi, "Pharmacokinetics of Rytary $^{\circledR}$, an extended-release capsule formulation of Carbidopa-Levodopa," Clinical Pharmacokinetics, vol. 56, no. 9, pp. 999-1014, 2017.

[75] D. M. de Matos, M. R. Viana, M. C. de Oliveira Alvim et al., "Pharmacokinetic profile and oral bioavailability of Kaurenoic acid from Copaifera spp. in rats," Fitoterapia, vol. 128, pp. 142-147, 2018.

[76] A. Hodge and B. Sterner, Toxicity Classes, Canadian Center for Occupational Health and Safety, 2005, https://www.ccohs.ca/ http://www.ccohs.ca/oshanswers/chemicals/id50.htm.

[77] M. Ahmed, "Acute toxicity (lethal dose 50 calculation) of herbal drug somina in rats and mice," Pharmacology \& Pharmacy, vol. 06, no. 03, pp. 185-189, 2015.

[78] R. B. Sandu, L. Tarțău, A. Miron, M. Zagnat, C. M. Ghiciuc, and C. E. Lupuşoru, "Experimental researches on acute toxicity of a Bidens tripartita extract in mice-preliminary investigation," Revista medico-chirurgicala a Societatii de Medici si Naturalisti din Iasi, vol. 116, no. 4, 2012. 


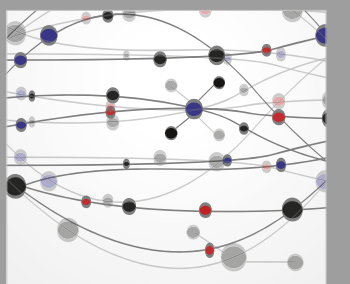

The Scientific World Journal
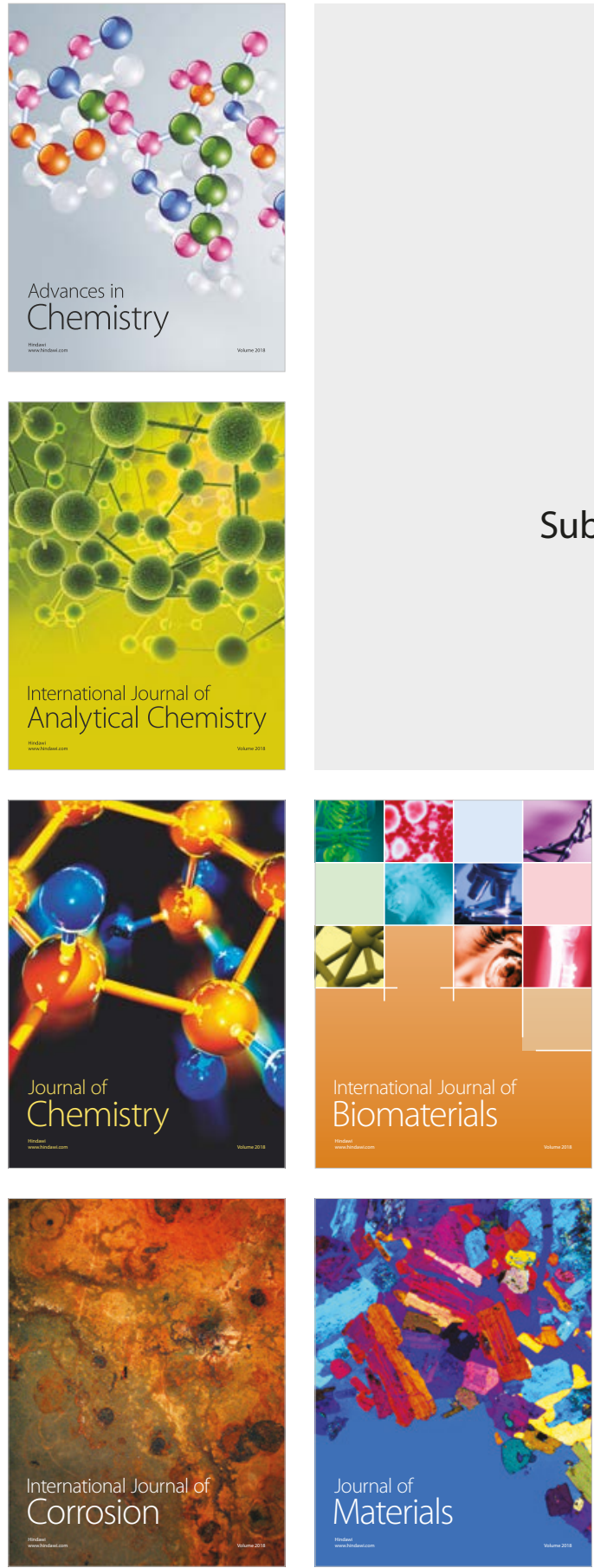

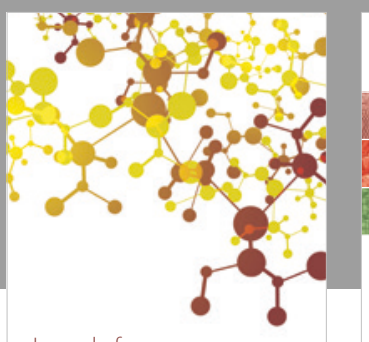

Journal of

Applied Chemistry
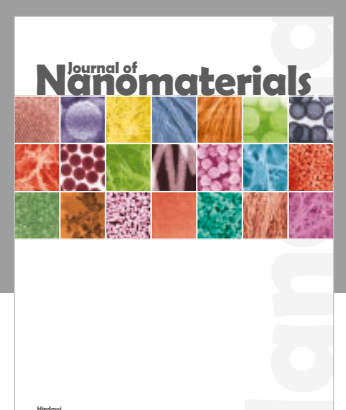

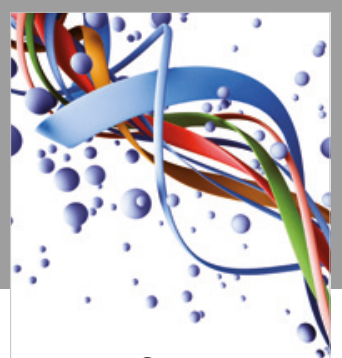

Scientifica

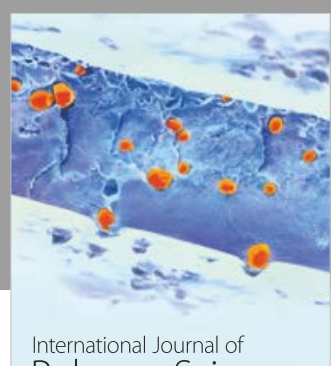

Polymer Science

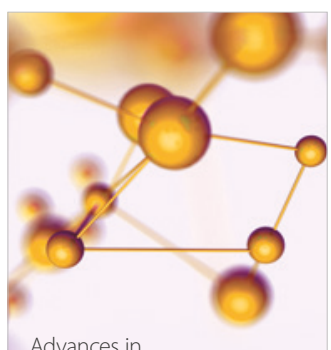

Physical Chemistry
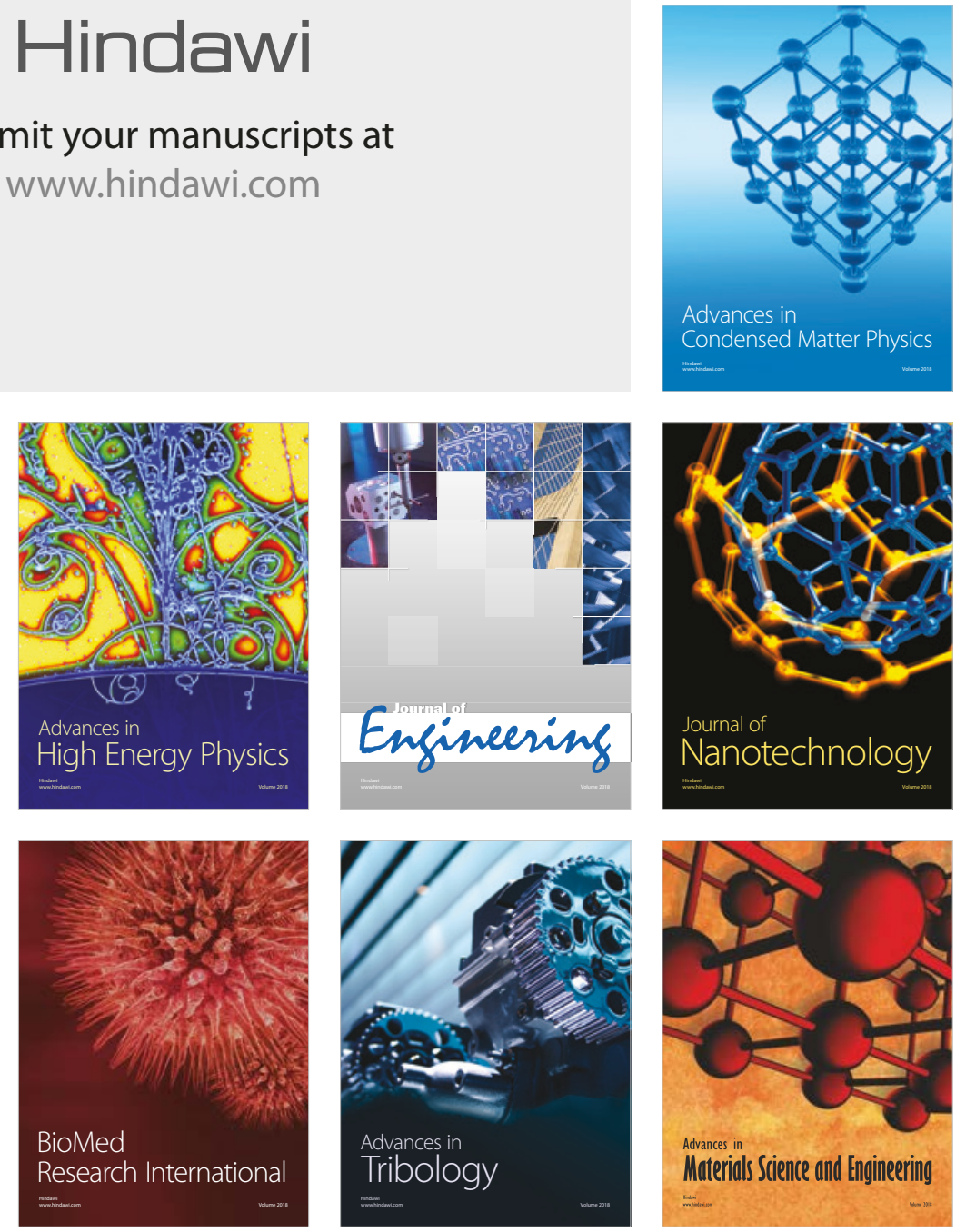